\title{
Panchromatic Absorption and Oxidation of an Iron(II) Spin Crossover Complex
}

Johannes Moll, ${ }^{\dagger}$ Christoph Förster,,$^{\dagger}$ Alexandra König, ${ }^{\dagger}$ Luca M. Carrella, ${ }^{\dagger}$ Manfred Wagner, ${ }^{\dagger}$ Martin Panthöfer,,$^{\dagger}$ Angela Möller, ${ }^{\dagger}$ Eva Rentschler, ${ }^{\dagger}$ and Katja Heinze, ${ }^{*}$

${ }^{\dagger}$ Department of Chemistry, Johannes Gutenberg University, Duesbergweg 10-14, 55128 Mainz, Germany; katja.heinze@uni-mainz.de

\$ Max Planck Institute for Polymer Research, Ackermannweg 10, 55128 Mainz, Germany.

\section{Iron(II) complexes}

Characterization of $\mathbf{1}[\mathbf{O T f}]_{2}\left(\mathrm{IR},{ }^{1} \mathrm{H}\right.$ NMR, ESI ${ }^{+}$) [Figures S1-S4]

Variable temperature NMR spectroscopic studies of 1[OTf] 2 [Figures S5-S9]

TDDFT and charge transfer analyses of $\mathbf{1}^{2+}$ [Figures S10-S12]

Variable temperature UV/Vis spectroscopic studies of 1[OTf]2 [Figures S13-S14]

Variable temperature magnetic susceptibility measurement of 1[OTf] 2 [Figure S15]

Square-wave voltammograms of of $\mathbf{1}[\mathbf{O T f}]_{2}$ [Figure S16]

\section{Iron(III) complex}

Characterization of $\mathbf{1}\left[\mathbf{P F}_{6}\right]_{3}\left({ }^{1} \mathrm{H}\right.$ NMR, EPR, IR, ESI $\left.{ }^{+}\right)$[Figures S17-S20]

TDDFT and charge transfer analysis of $\mathbf{1}^{3+}$ [Figure S21] 


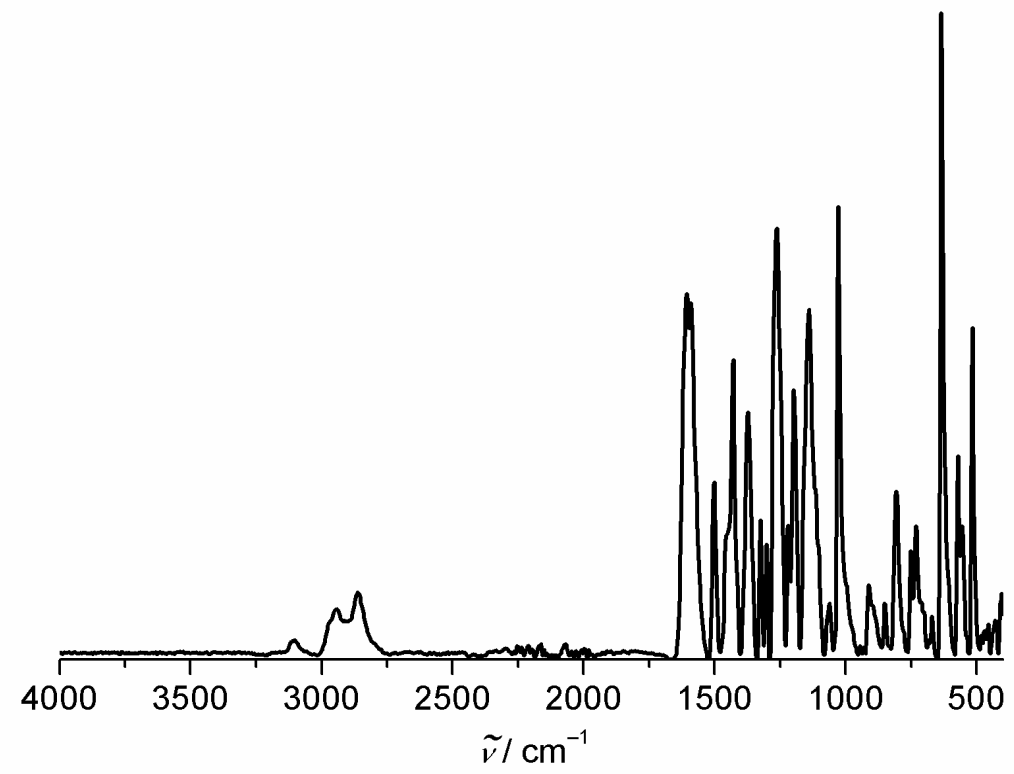

Figure S1. ATR IR spectrum of 1[OTf]2.

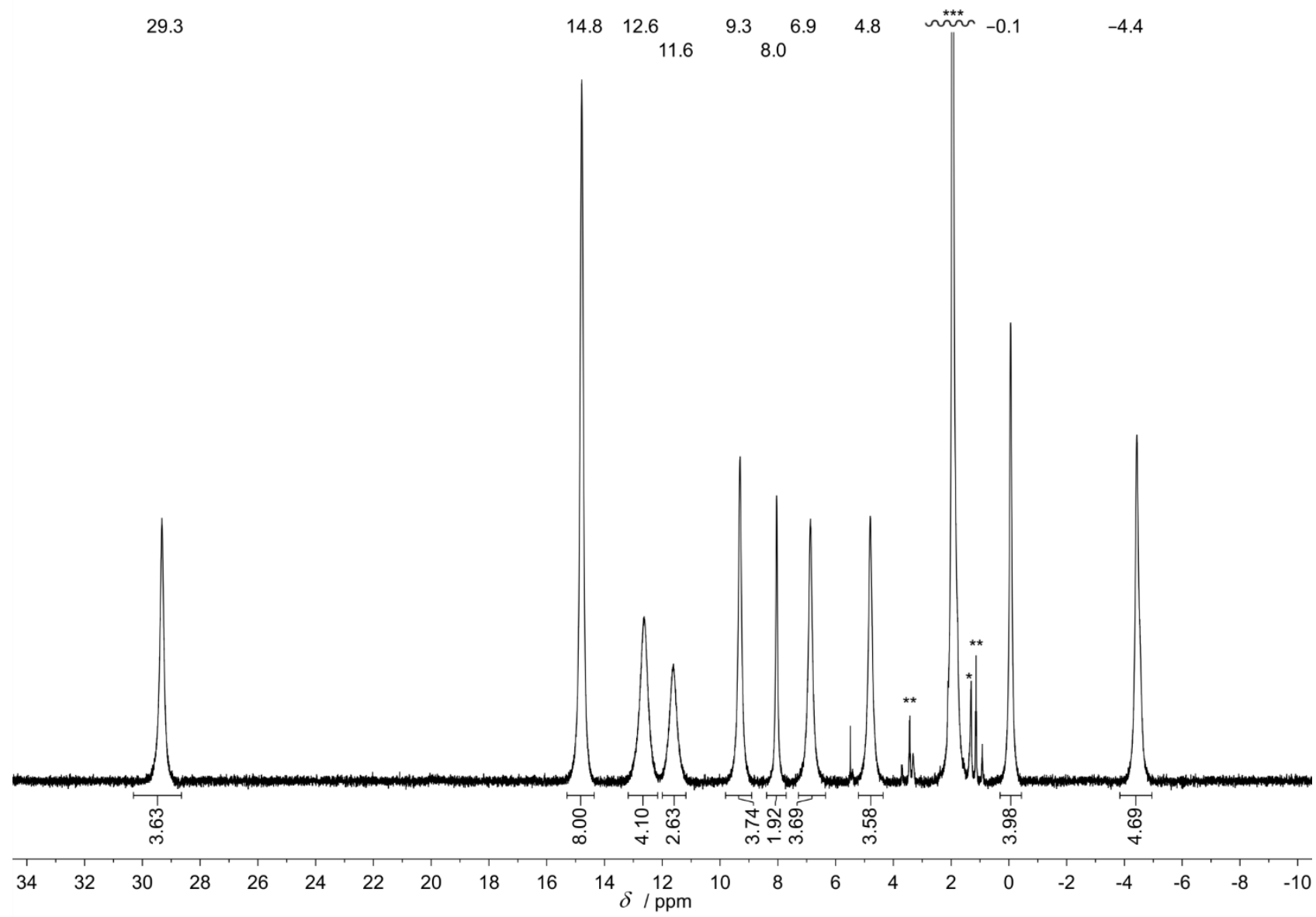

Figure S2. ${ }^{1} \mathrm{H}$ NMR spectrum of $\mathbf{1}$ [OTf] $]_{2}$ in dry, deaerated $\mathrm{CD}_{3} \mathrm{CN}$ at $293 \mathrm{~K}$ (* residual grease; ** residual diethylether; $* * *$ acetonitrile). 


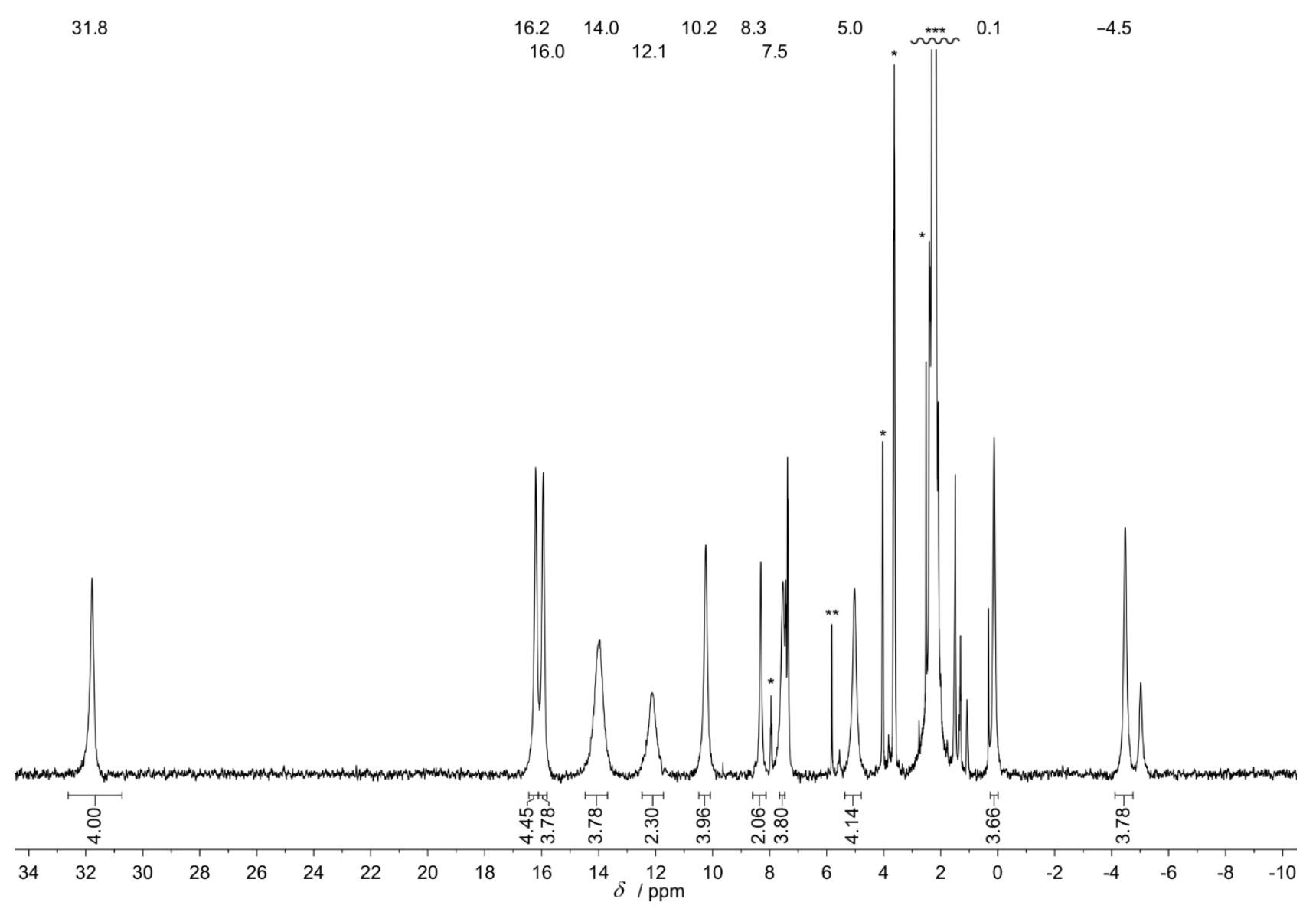

Figure S3. ${ }^{1} \mathrm{H}$ NMR spectrum of $\mathbf{1}[\text { OTf }]_{2}$ in dry, deaerated $\mathrm{d}_{6}$-acetone at $293 \mathrm{~K}$ (* residual $[\mathrm{dgpyH}]^{+} ; * *$ residual dichlormethane; *** acetone), chemical shifts referenced to the acetone resonance stemming from the capillary insert of the Evans' NMR measurement.

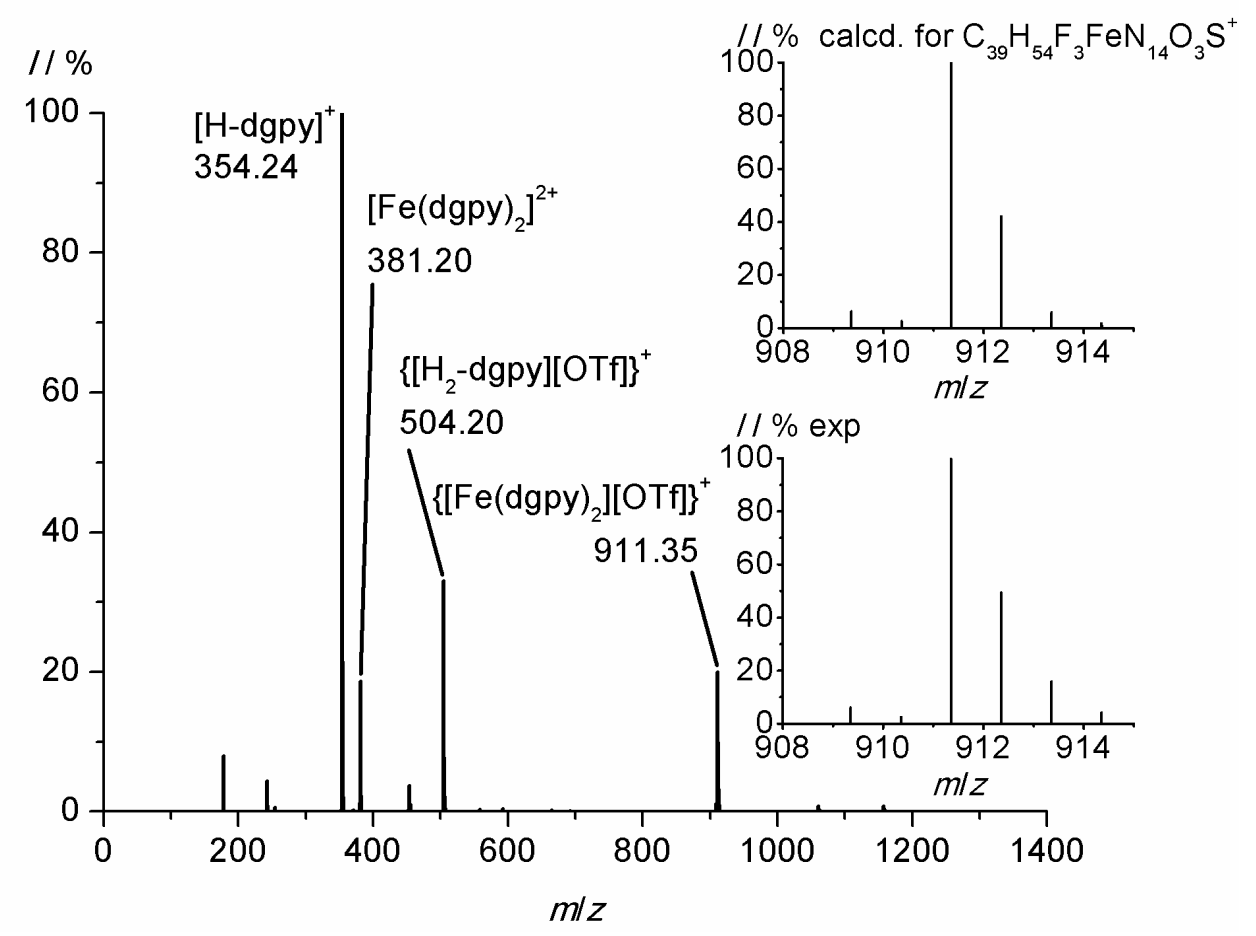

Figure S4. $\mathrm{ESI}^{+}$mass spectrum of $\mathbf{1}[\mathbf{O T f}]_{2}$ in dry, deaerated acetonitrile. 


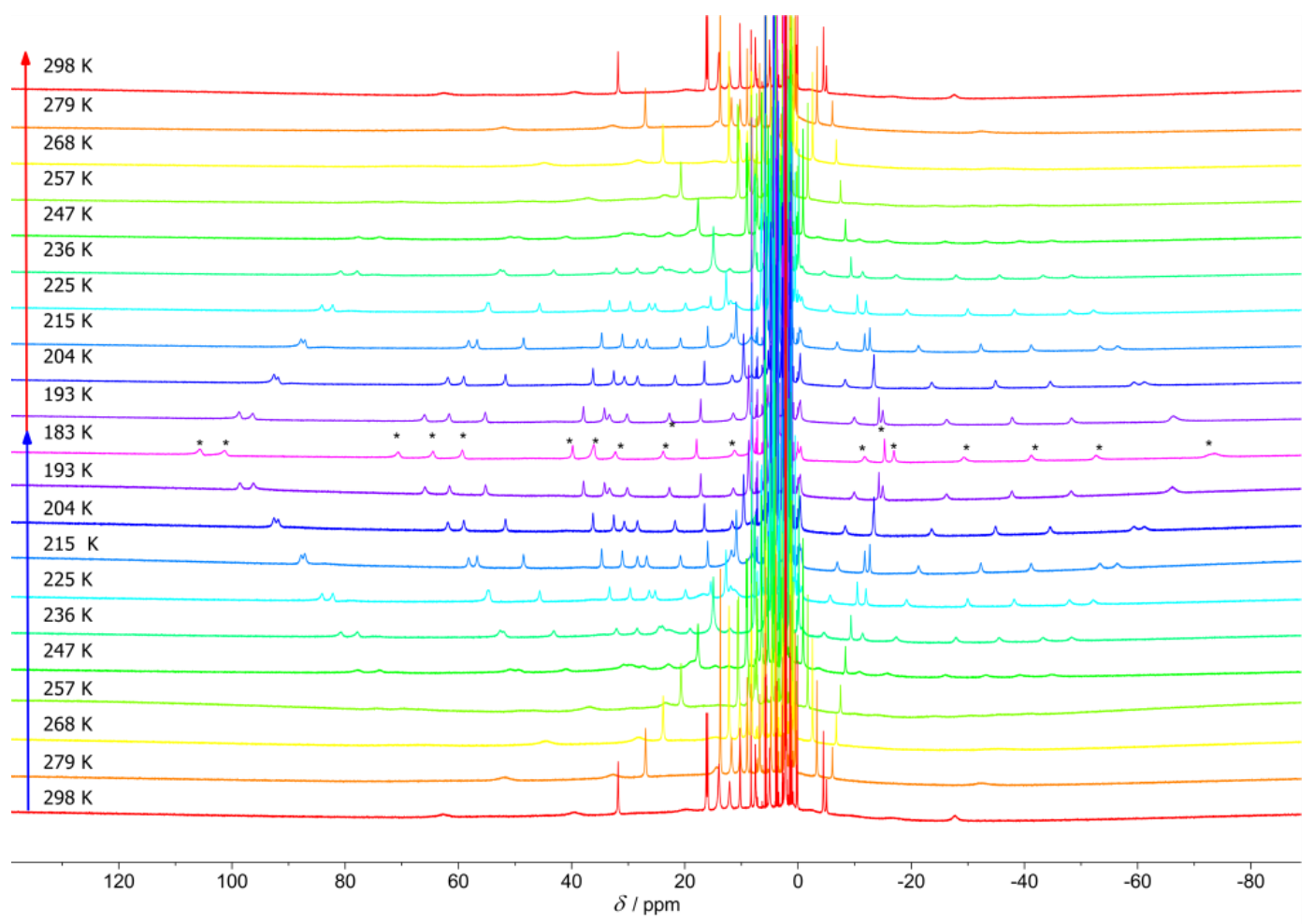

Figure S5. Variable temperature ${ }^{1} \mathrm{H}$ NMR spectra of $\mathbf{1}$ [OTf $]_{2}$ in $\mathrm{d}_{6}$-acetone (cooling and re-warming).

Resonances of the cis-fac isomer are marked with asterisks in the spectrum at $183 \mathrm{~K}$.

Table S1. $k_{\mathrm{hs}} / \mathrm{s}^{-1}$ calculated from temperature dependent ${ }^{1} \mathrm{H}$ NMR experiments using the following equation:

$k_{\mathrm{hl}}(T)=\frac{\pi}{2}\left(\frac{\left(\delta_{\mathrm{hs}}-\delta_{\mathrm{ls}}\right)^{2}}{\Delta(T)}\right)$

with $\delta_{\text {ss }}$ and $\delta_{\text {s }}$ denoting the chemical shifts of the same resonance for the high-spin and low-spin state, respectively, and $\Delta(T)$ is the line width of the respective resonance.

\begin{tabular}{cccc}
\hline$T / \mathrm{K}$ & $\begin{array}{c}\text { resonance at } \\
\delta=31.8 \mathrm{ppm}\end{array}$ & $\begin{array}{c}\text { resonance at } \\
\delta=16.2 \mathrm{ppm}\end{array}$ & $\begin{array}{c}\text { resonance at } \\
\delta=16.0 \mathrm{ppm}\end{array}$ \\
\hline 298 & $3.14 \times 10^{6}$ & $1.57 \times 10^{6}$ & $1.49 \times 10^{6}$ \\
279 & $2.58 \times 10^{6}$ & $1.16 \times 10^{6}$ & $1.10 \times 10^{6}$ \\
268 & $2.07 \times 10^{6}$ & & \\
257 & $1.81 \times 10^{6}$ & $8.29 \times 10^{5}$ & $7.89 \times 10^{5}$ \\
247 & $1.52 \times 10^{6}$ & $7.54 \times 10^{5}$ & $7.17 \times 10^{5}$ \\
236 & $1.31 \times 10^{6}$ & $7.74 \times 10^{5}$ & $7.36 \times 10^{5}$ \\
225 & $1.49 \times 10^{6}$ & $8.29 \times 10^{5}$ & $7.89 \times 10^{5}$ \\
215 & $1.52 \times 10^{6}$ & $9.68 \times 10^{5}$ & $9.21 \times 10^{5}$ \\
204 & $1.69 \times 10^{6}$ & $1.16 \times 10^{6}$ & $1.10 \times 10^{6}$ \\
193 & $2.00 \times 10^{6}$ & $1.61 \times 10^{6}$ & $1.53 \times 10^{6}$ \\
\hline
\end{tabular}




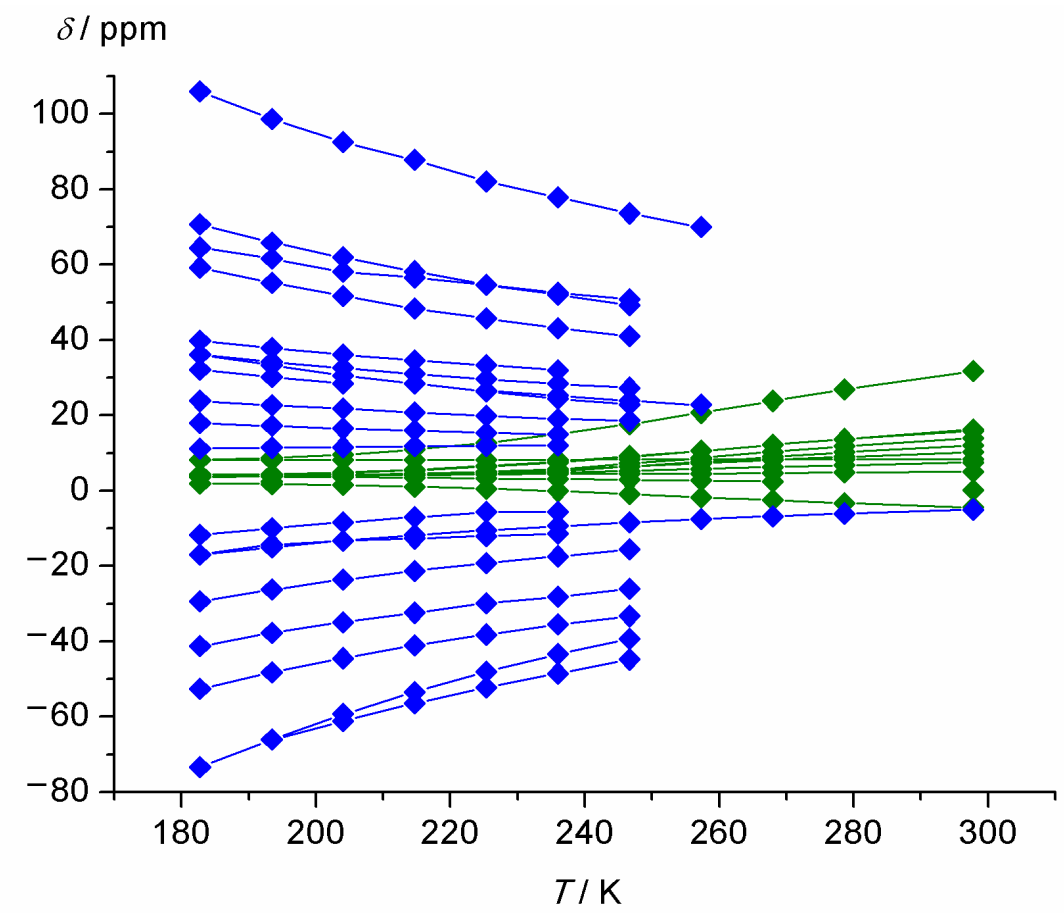

Figure S6. Chemical shifts of ${ }^{1} \mathrm{H}$ NMR resonances of $\mathbf{1}[\mathbf{O T f}]_{2}$ vs. temperature derived from variable temperature ${ }^{1} \mathrm{H}$ NMR experiments in dry, deaerated d6-acetone, green: $\boldsymbol{m e r}-\mathbf{1}^{2+}$, blue: $\boldsymbol{c i s}$ fac $-\mathbf{1}^{2+}$. 
a)
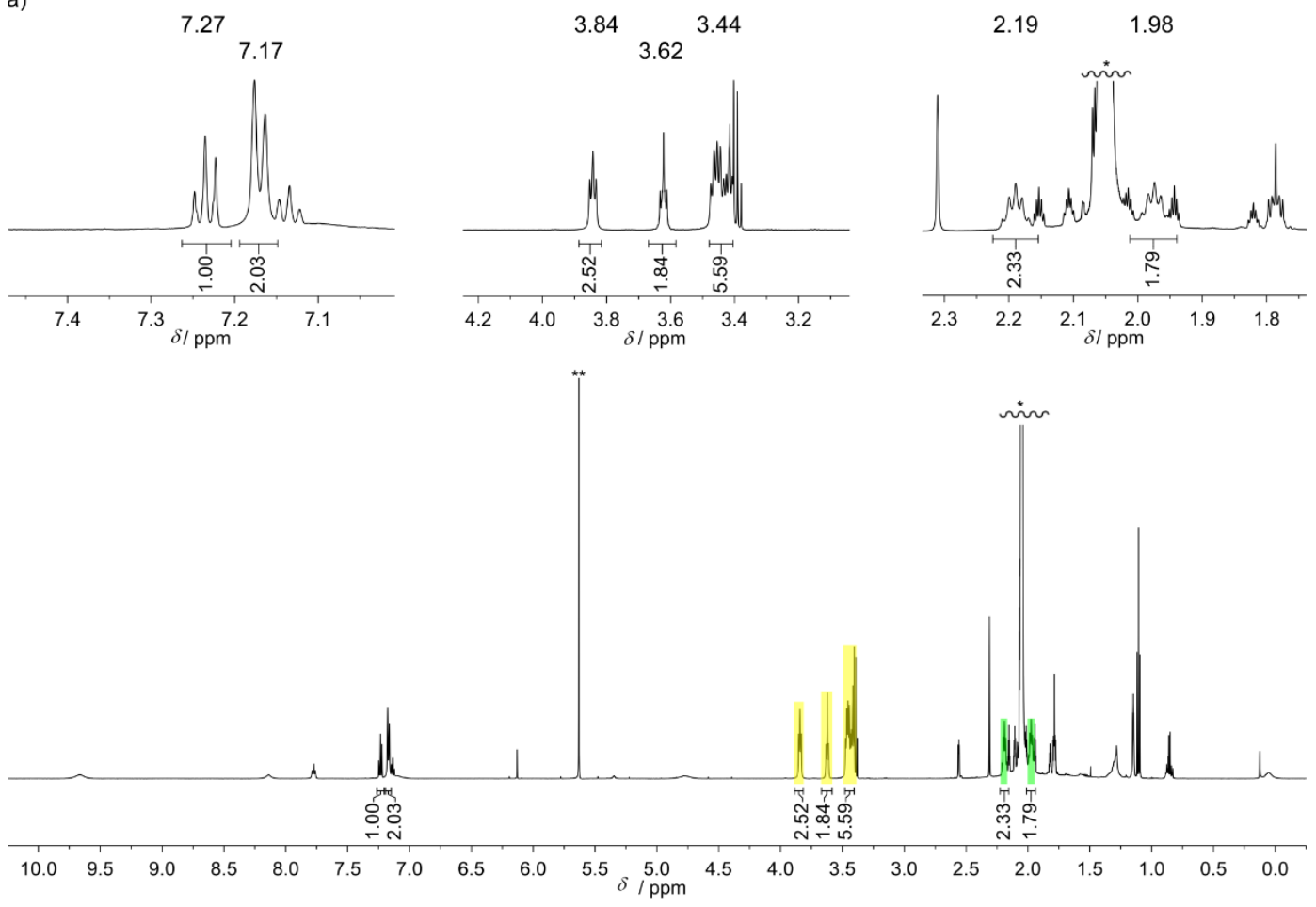

b)

$$
7.31
$$$$
\begin{array}{cc}
3.83 & 3.30 \\
& 3.22 \\
& 3.16
\end{array}
$$

1.98

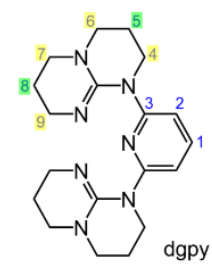

dgpy

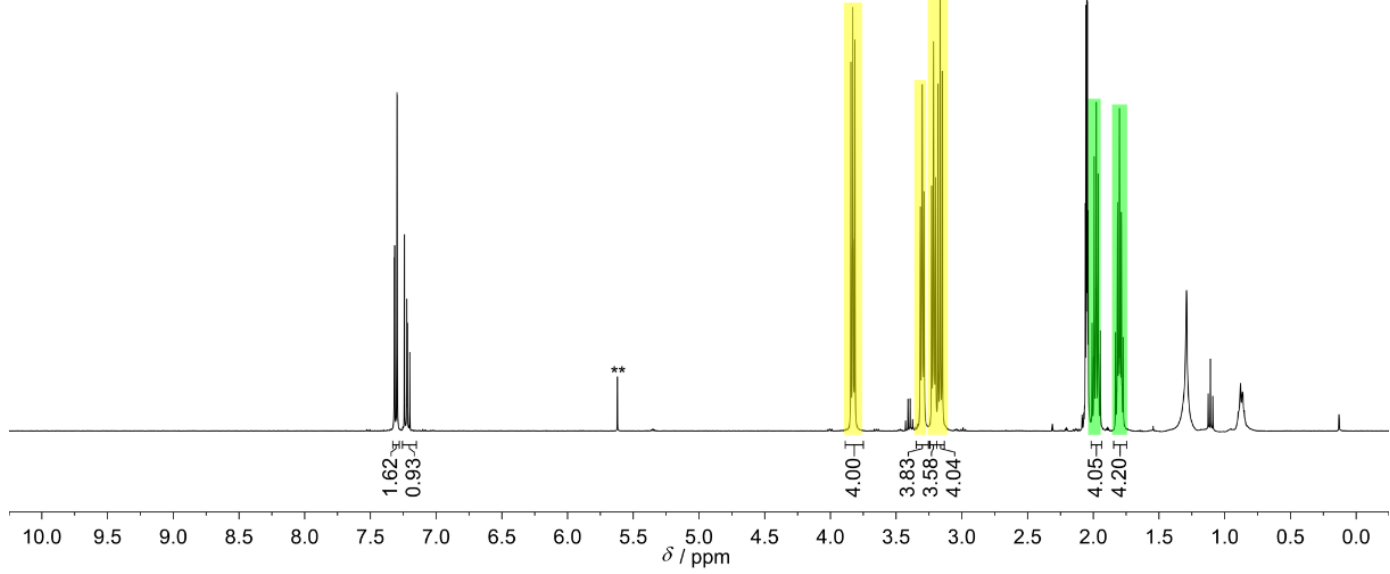

Figure S7. ${ }^{1} \mathrm{H}$ NMR spectra of a) 1[OTf] 2 after 7 days in $\mathrm{d}_{6}$-acetone at $293 \mathrm{~K}$ showing resonances of the free ligand with reduced integrals for $\mathrm{H}^{4}-\mathrm{H}^{9}$ resulting from $\mathrm{H} / \mathrm{D}$ exchange and $b$ ) dgpy in in dry, deaerated $\mathrm{d}_{6}$-acetone at $293 \mathrm{~K}$ for comparison (* acetone; ** residual dichloromethane). 


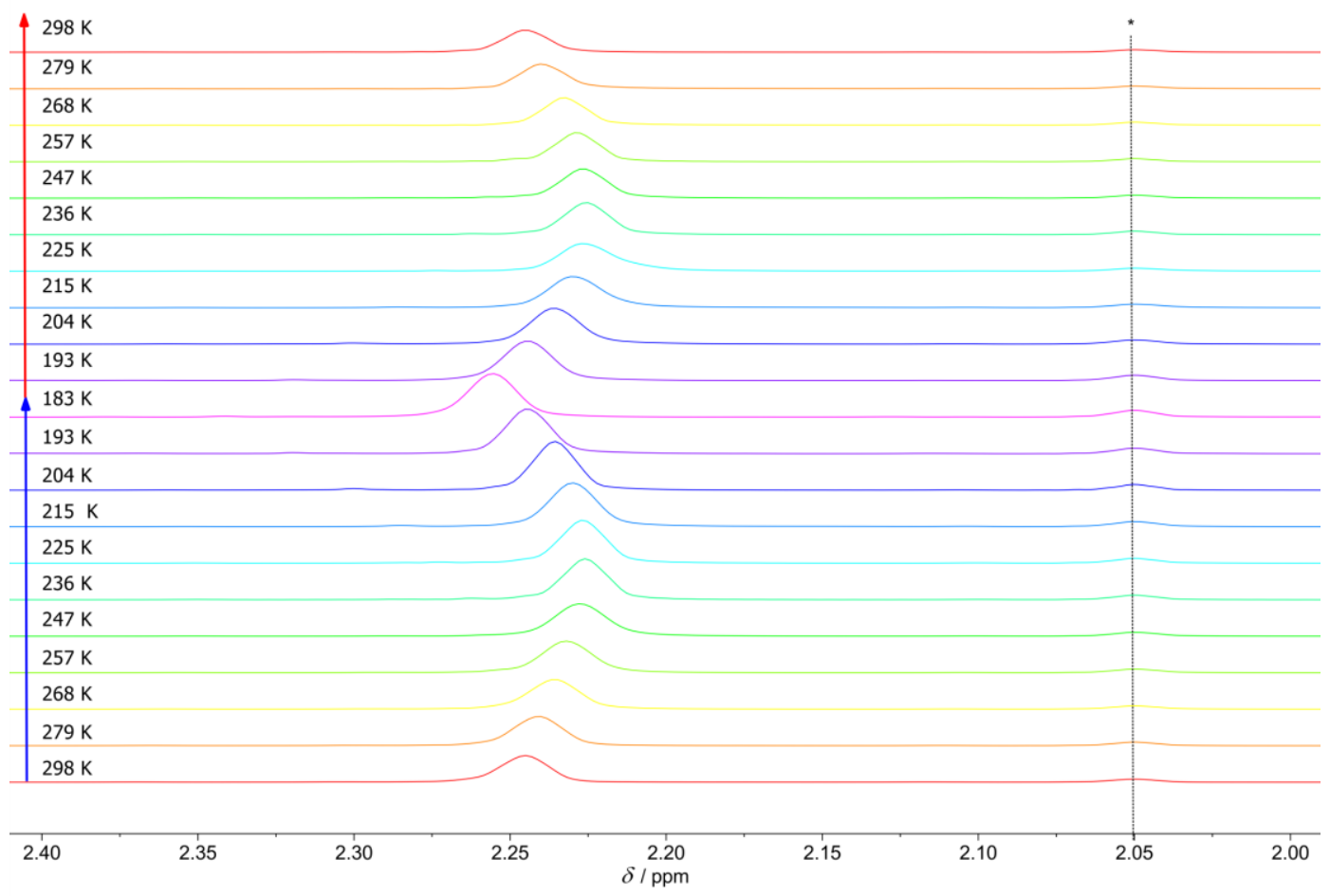

Figure S8. Excerpt of ${ }^{1} \mathrm{H}$ NMR spectra of $\mathbf{1}[\mathbf{O T f}]_{2}$ in dry, deaerated d6-acetone at different temperatures showing the temperature dependent shift of the acetone resonance referenced to the acetone standard in the inside capillary (Evans' method).

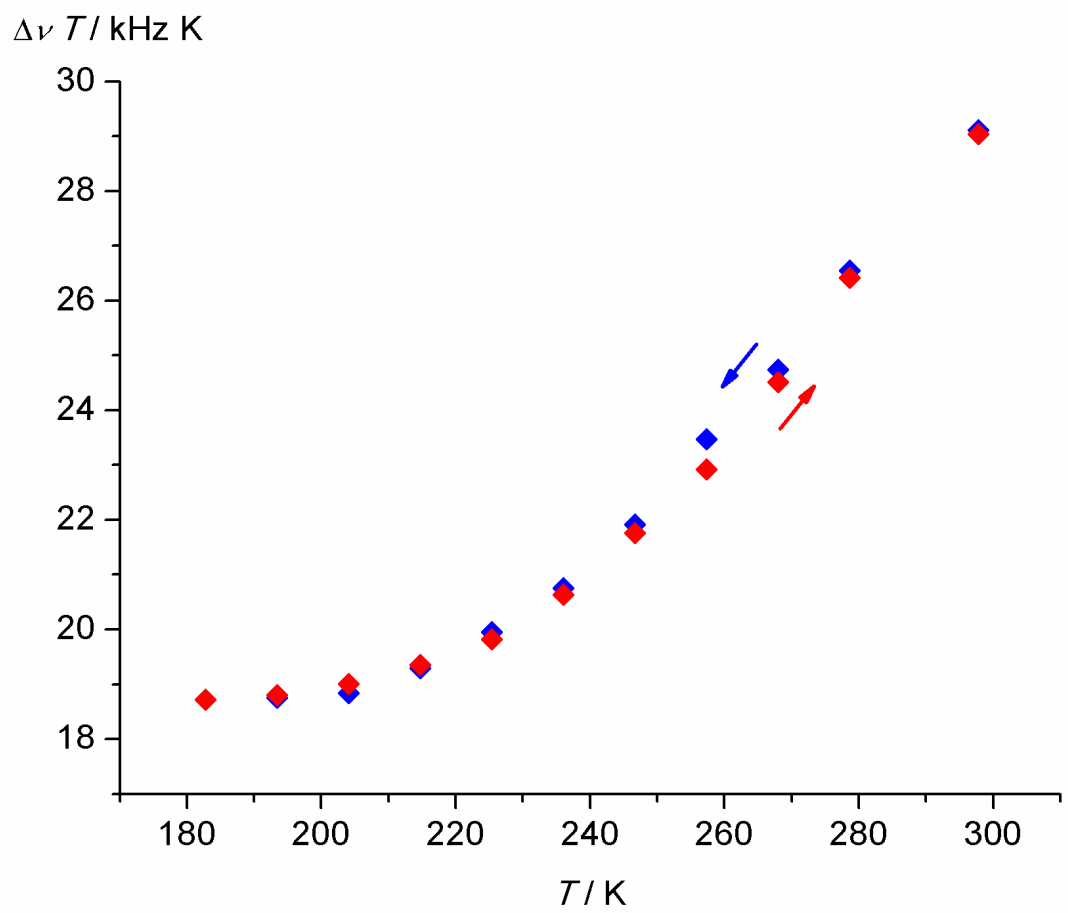

Figure S9. $\Delta v T$ vs. $T$ data obtained from ${ }^{1} \mathrm{H}$ NMR measurements of 1[OTf] $]_{2}$ using Evans' method in d6-acetone (blue: cooling; red: re-warming). 
a)

\section{CT number}

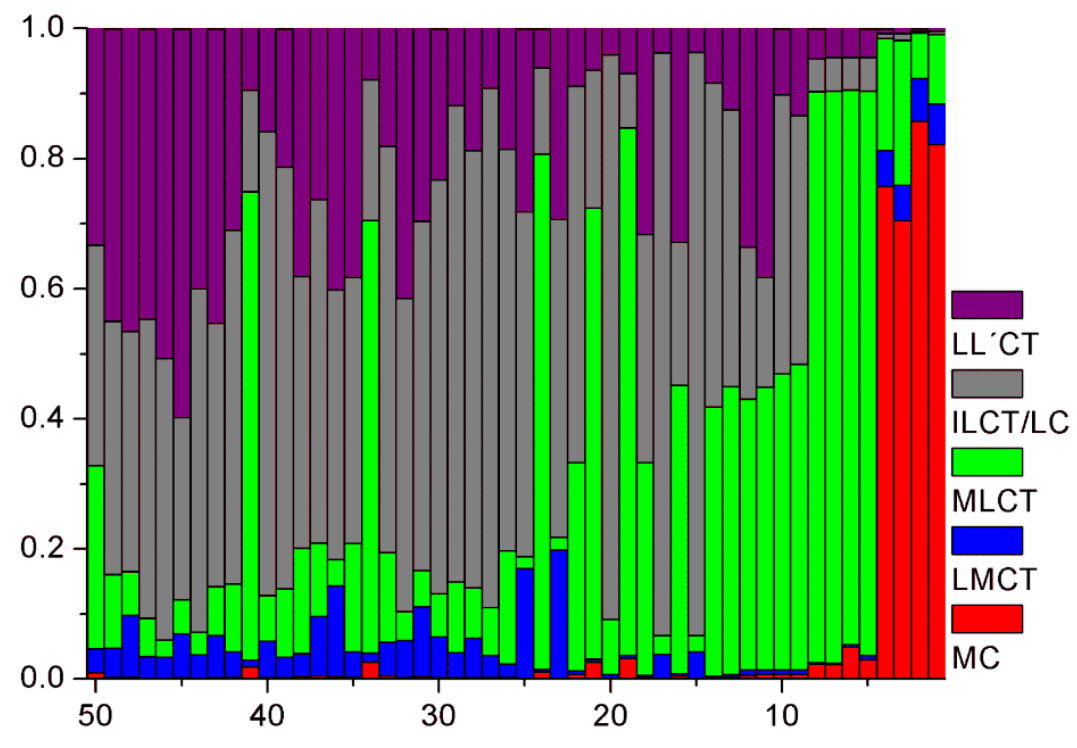

b)

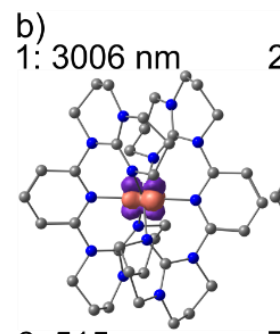

6: $515 \mathrm{~nm}$

state

$4: 646 \mathrm{~nm}$

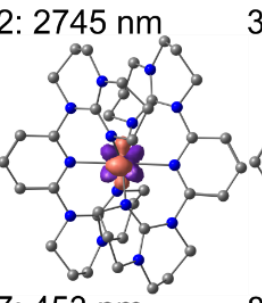

3: $798 \mathrm{~nm}$
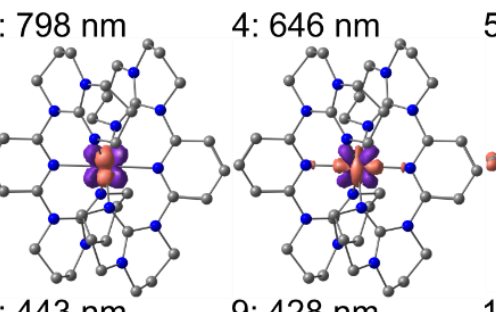

5: $545 \mathrm{~nm}$
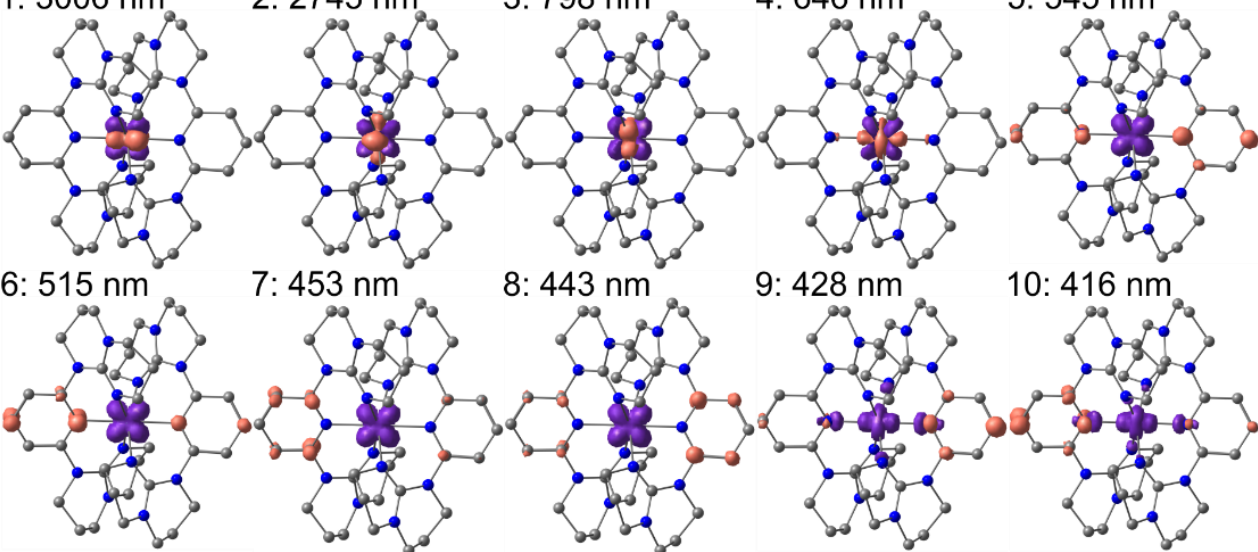

7: $453 \mathrm{~nm}$

8: $443 \mathrm{~nm}$

9: $428 \mathrm{~nm}$

10: $416 \mathrm{~nm}$
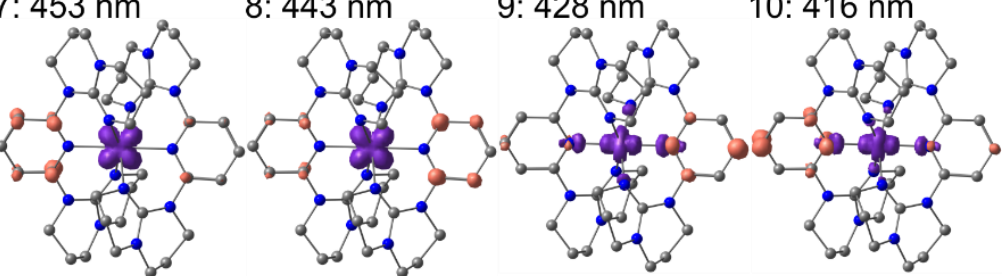

11: $390 \mathrm{~nm}$

12: $381 \mathrm{~nm}$
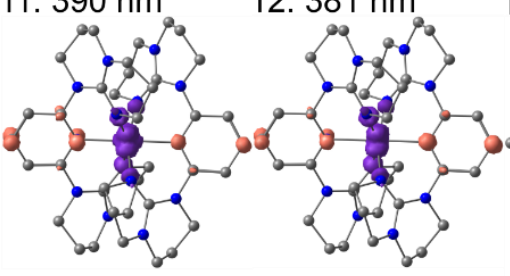

13: $356 \mathrm{~nm}$

14: $353 \mathrm{~nm}$

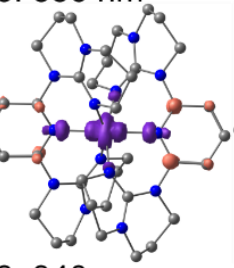

90

15: $346 \mathrm{~nm}$
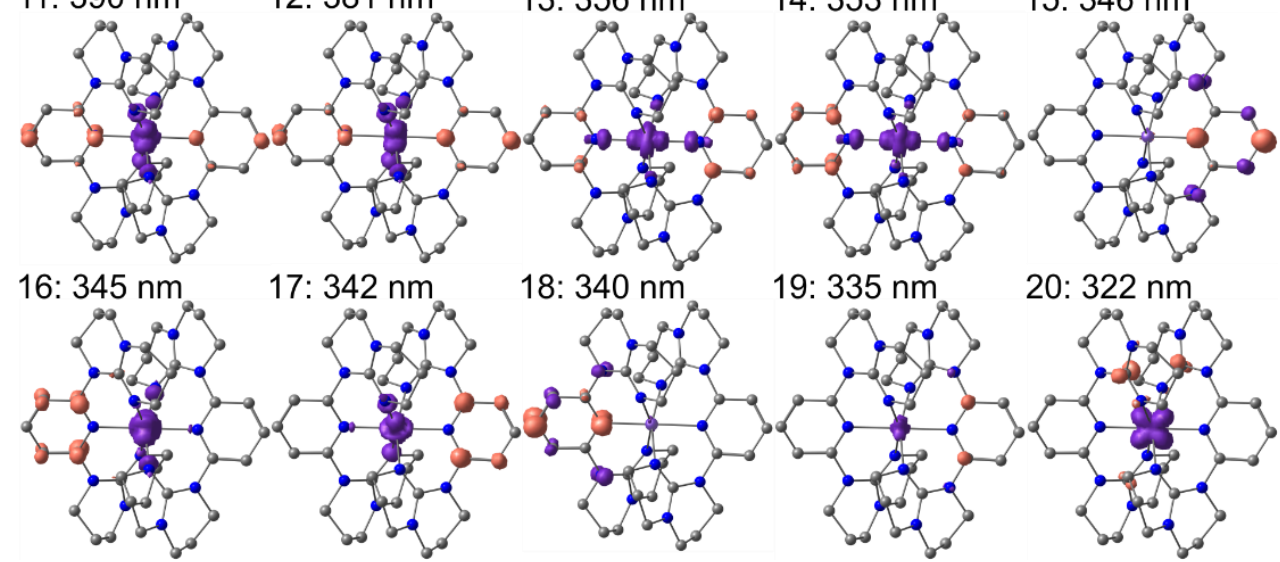

17: $342 \mathrm{~nm}$

18: $340 \mathrm{~nm}$

19: $335 \mathrm{~nm}$

20: $322 \mathrm{~nm}$
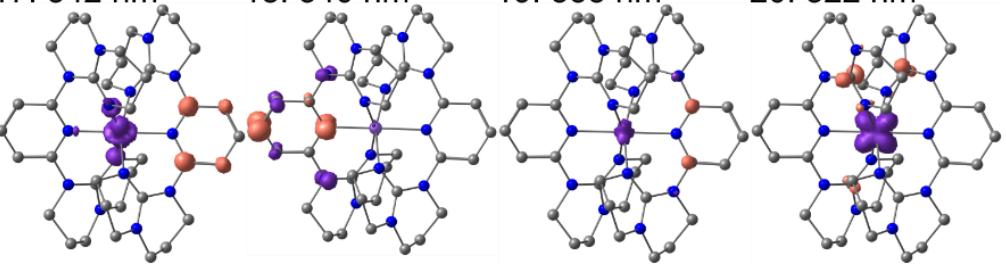

Figure S10. a) Charge transfer analysis of the 50 lowest spin-allowed transitions of high-spin $\boldsymbol{m e r}$ $\mathbf{1}^{\mathbf{2}}$ (red: MC, blue: LMCT, green: MLCT, gray: ILCT/LC, purple: LL'CT) and b) difference densities of the 20 lowest spin-allowed transitions of high-spin $\mathbf{m e r - \mathbf { 1 } ^ { 2 + }}$ with the respective wavelength (purple = electron loss; orange = electron gain). The two lowest transitions reflect the split ${ }^{5} \mathrm{~T}$ ground state. 
a)

\section{CT number}
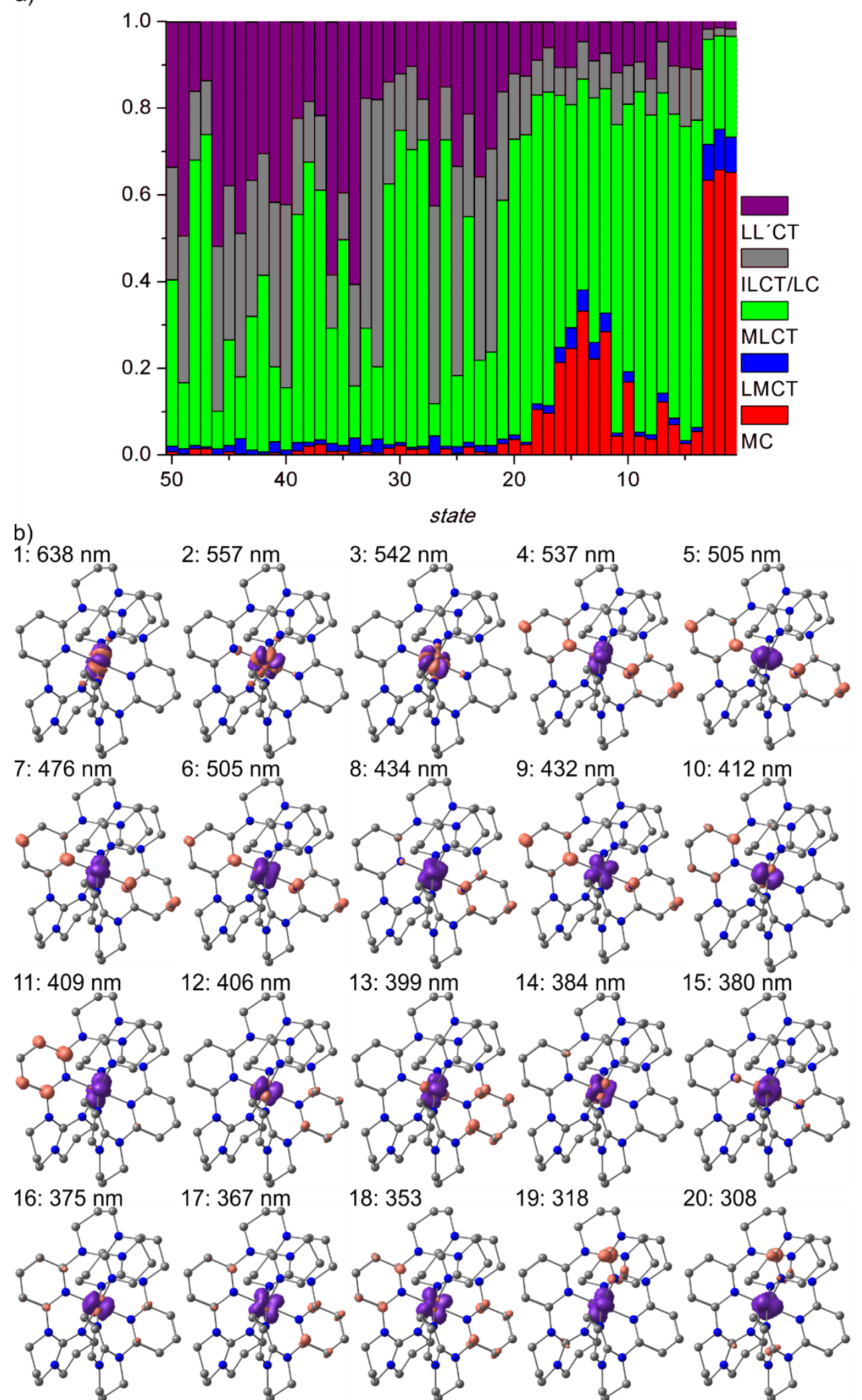

Figure S11. a) Charge transfer analysis of the 50 lowest spin-allowed transitions of low-spin $\boldsymbol{m e r -}$ $\mathbf{1}^{\mathbf{2 +}}$ (red: MC, blue: LMCT, green: MLCT, gray: ILCT/LC, purple: LL'CT) and b) difference densities of the 20 lowest spin-allowed transitions of low-spin $\mathbf{m e r - \mathbf { 1 } ^ { 2 + }}$ with the respective wavelength (purple $=$ electron loss; orange $=$ electron gain). 
a)

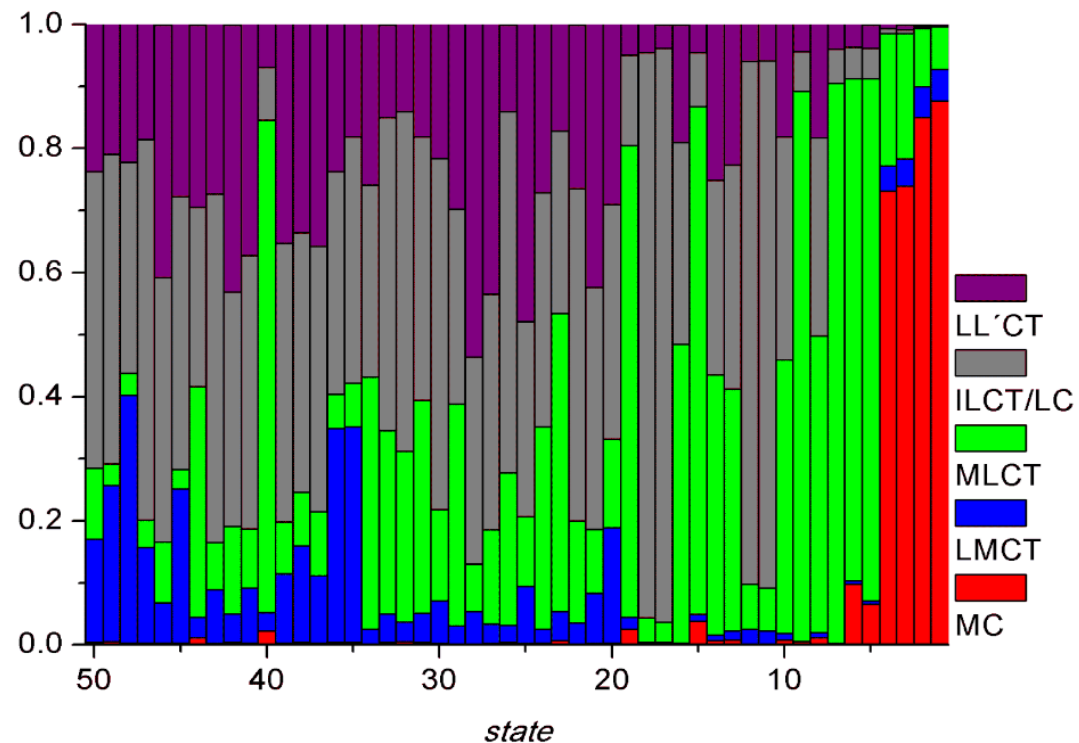

b)
1: $4058 \mathrm{~nm}$
2: $3038 \mathrm{~nm}$
3: $775 \mathrm{~nm}$
4: $728 \mathrm{~nm}$
5: $521 \mathrm{~nm}$
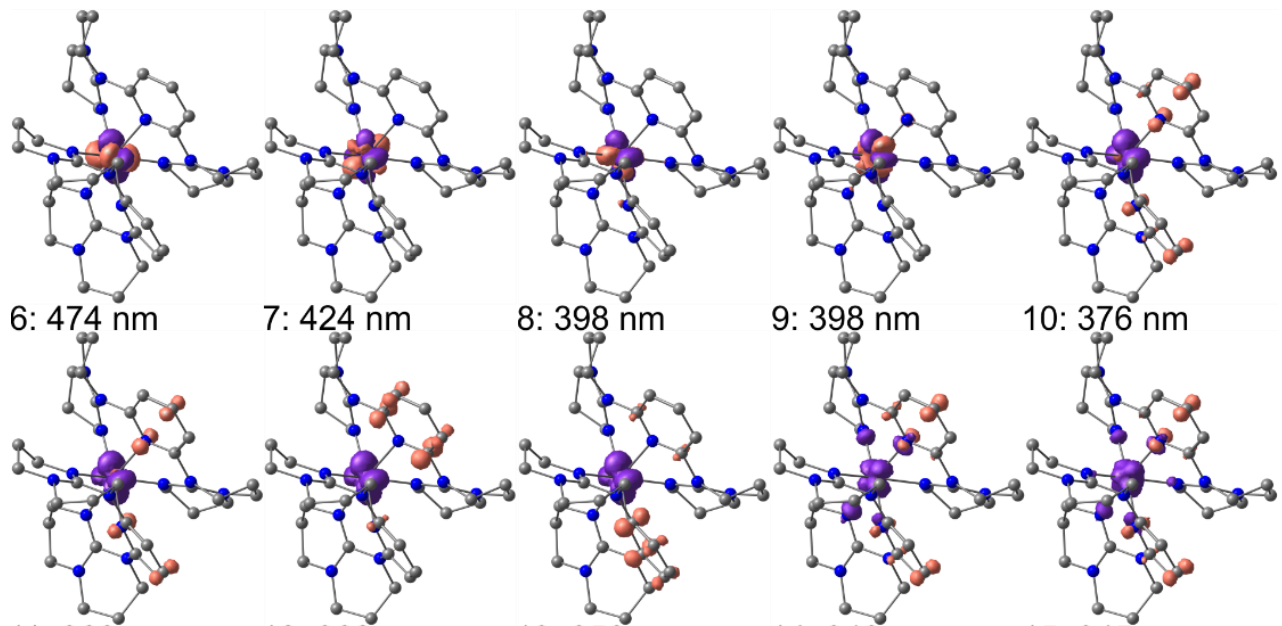

7: $424 \mathrm{~nm}$

8: $398 \mathrm{~nm}$

9: $398 \mathrm{~nm}$
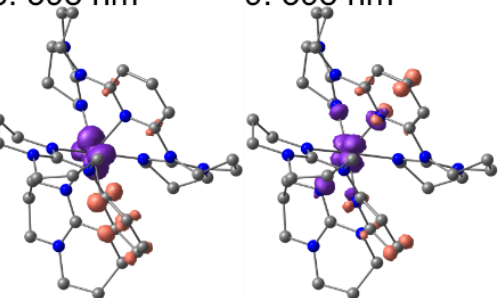

10: $376 \mathrm{~nm}$

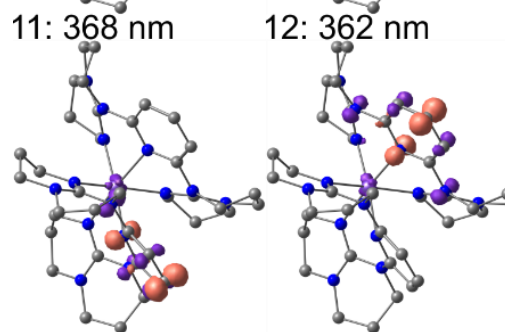

13: $358 \mathrm{~nm}$
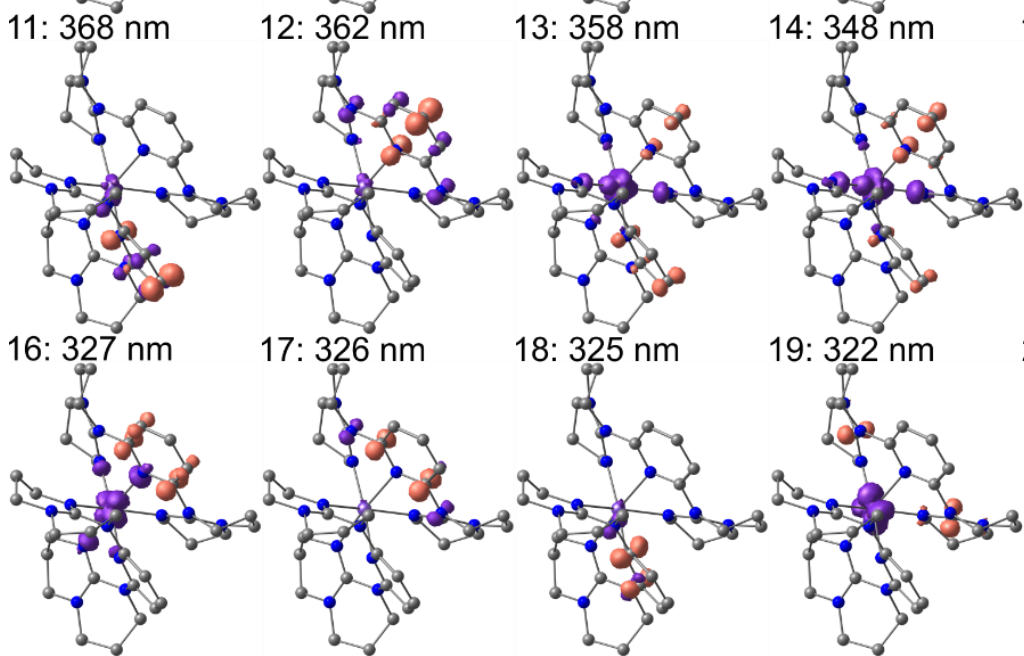

18: $325 \mathrm{~nm}$
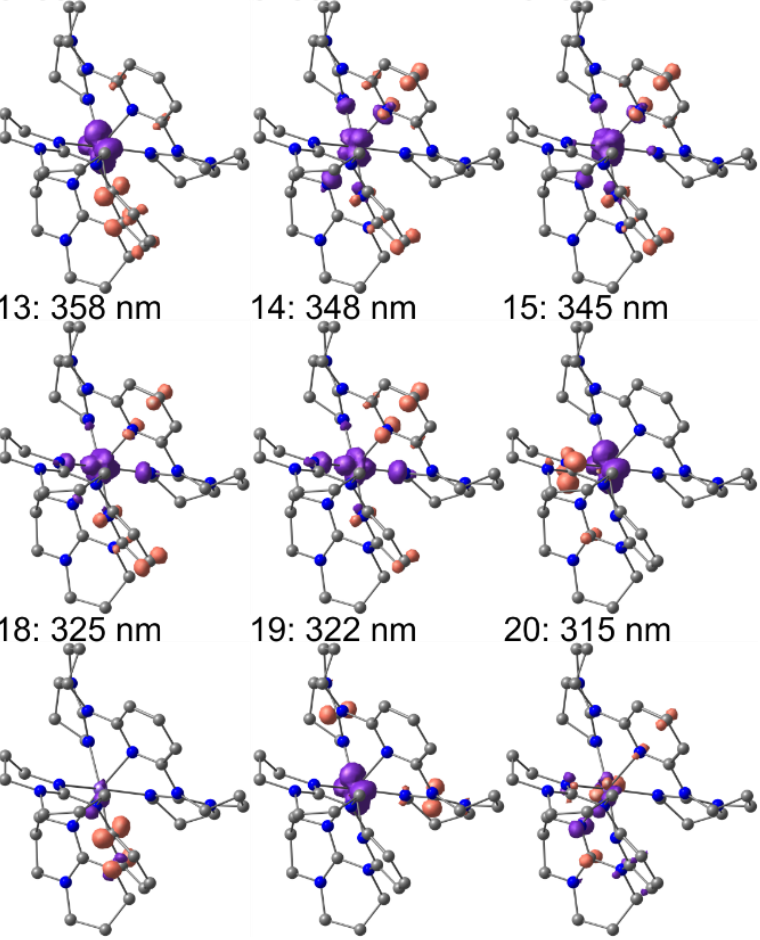

15: $345 \mathrm{~nm}$

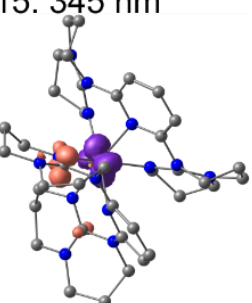

20: $315 \mathrm{~nm}$

Figure S12. a) Charge transfer analysis of the 50 lowest spin-allowed transitions of high-spin cis$f a c-\mathbf{1}^{2+}$ (red: MC, blue: LMCT, green: MLCT, gray: ILCT/LC, purple: LL'CT) and b) difference densities of the 20 lowest spin-allowed transitions of high-spin cis-fac-1 ${ }^{2+}$ with the respective wavelength (purple $=$ electron loss; orange $=$ electron gain). The two lowest transitions reflect the split ${ }^{5} \mathrm{~T}$ ground state. 
a) $\Delta \varepsilon / \mathrm{I} \mathrm{mol}^{-1} \mathrm{~cm}^{-1}$

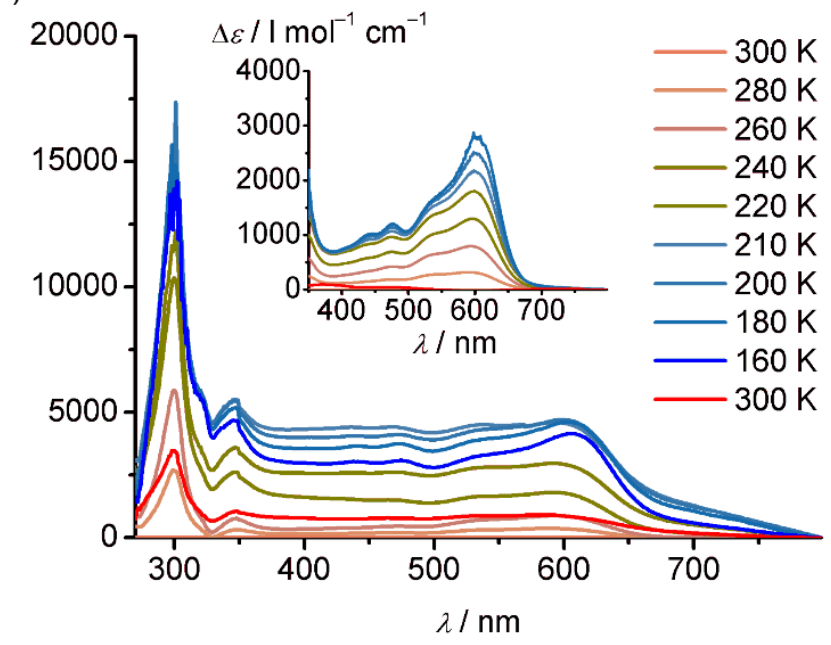

b) $\Delta \varepsilon / \mathrm{I} \mathrm{mol}^{-1} \mathrm{~cm}^{-1}$

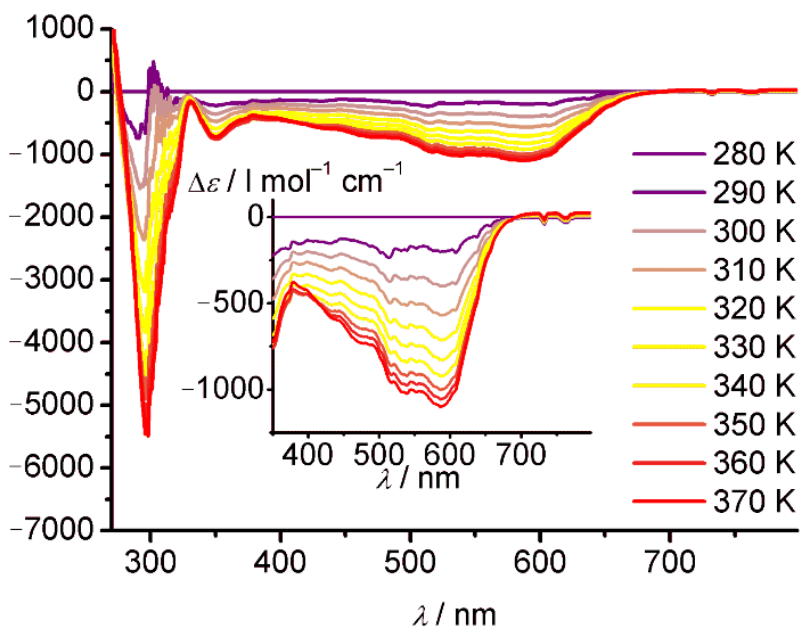

c)

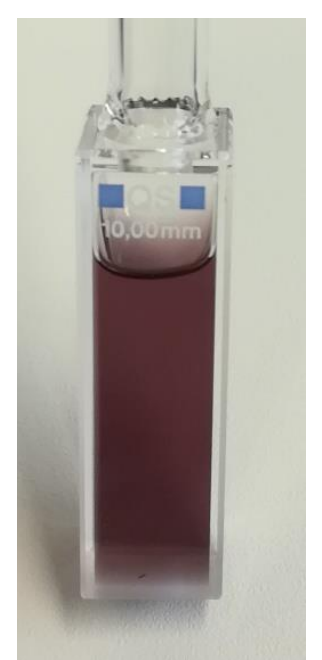

Figure S13. Variable temperature differential UV/Vis spectra of 1[OTf $]_{2}$ in butyronitrile, a) during cooling from $300 \mathrm{~K}$ to $160 \mathrm{~K}$ and re-warming to $300 \mathrm{~K}$ and $\mathrm{b}$ ) during warming from $280 \mathrm{~K}$ to $370 \mathrm{~K}$. c) Photograph of a dilute $\mathrm{CH}_{3} \mathrm{CN}$ solution of $\mathbf{1}[\mathbf{O T f}]_{2}$. 
a) $\quad \varepsilon_{540} / \mathrm{I} \mathrm{mol}^{-1} \mathrm{~cm}^{-1}$

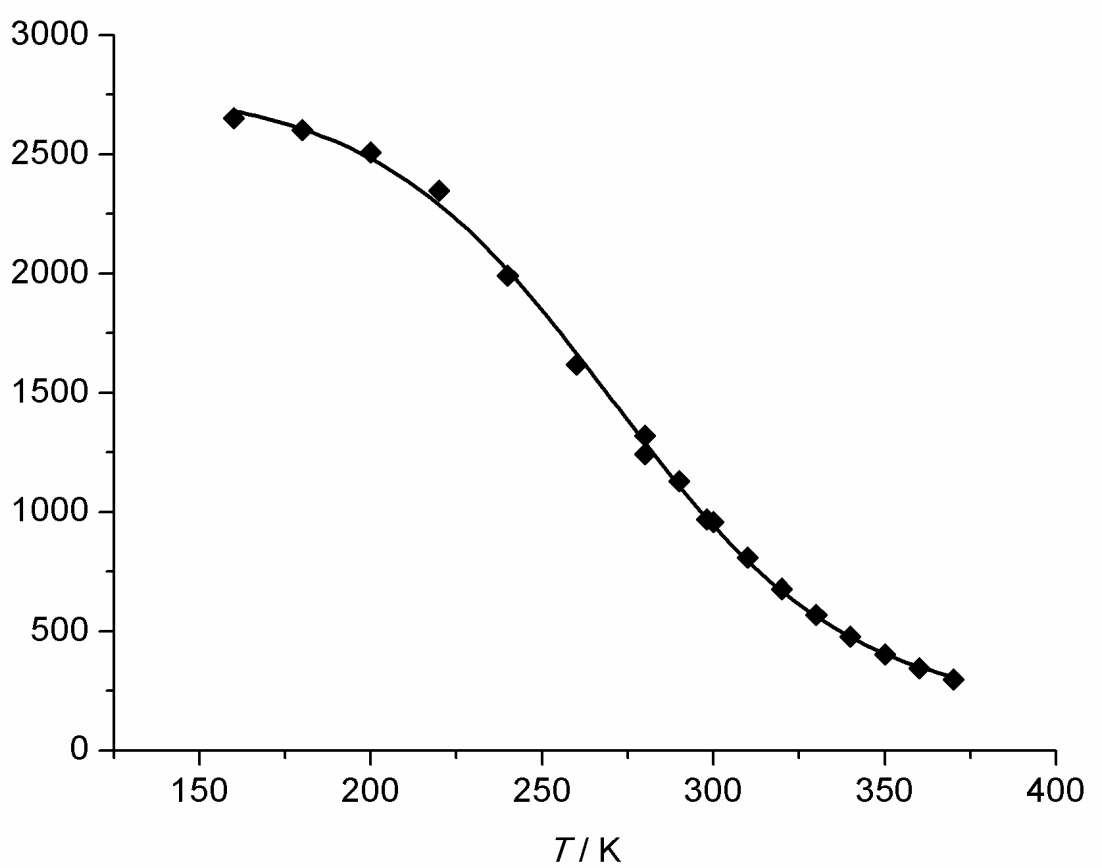

b) Abs.

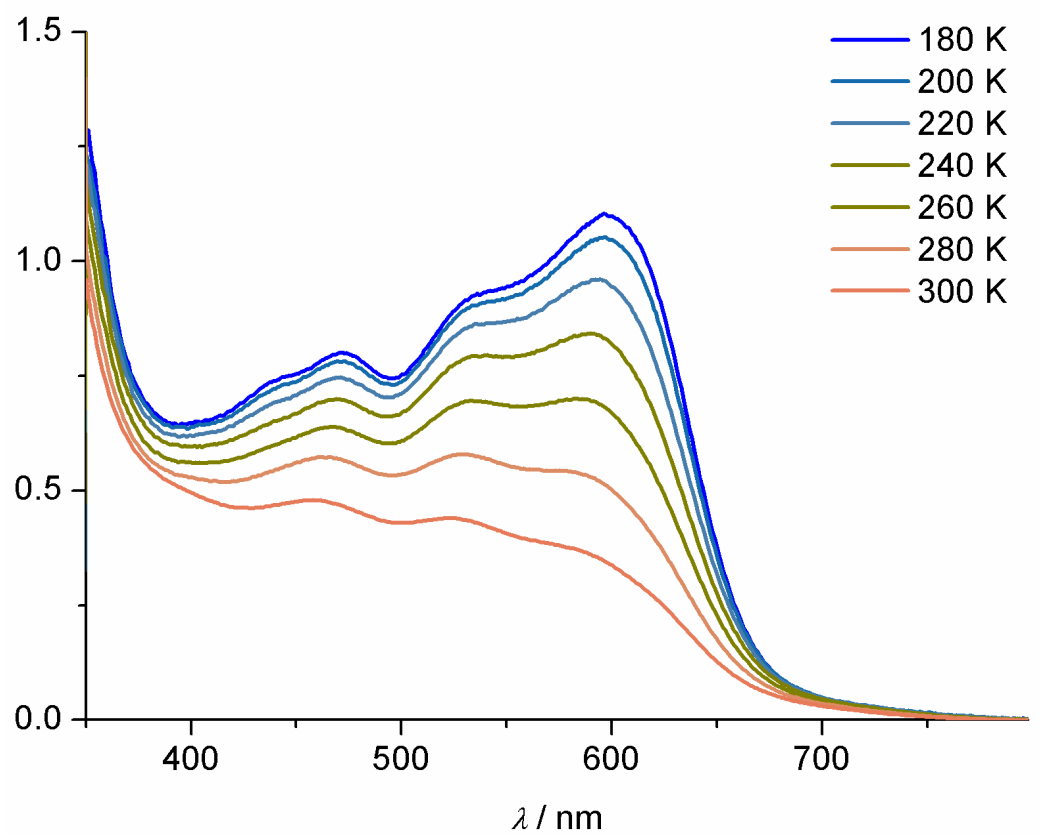

Figure S14. a) $\varepsilon_{540}$ vs. $T$ data obtained from optical measurements of $\mathbf{1}[\mathbf{O T f}]_{2}$ in butyronitrile and according to $\mathrm{R} \ln K=-\Delta H / T+\Delta S$ using $\Delta H=30(1) \mathrm{kJ} \mathrm{mol}^{-1}$ and $\Delta S=107(3) \mathrm{J} \mathrm{mol}^{-1} \mathrm{~K}^{-1}$. b) Variable temperature $\mathrm{UV} / \mathrm{Vis}$ spectra of $\mathbf{1}[\mathbf{O T f}]_{2}$ in acetone during cooling to $180 \mathrm{~K}$ (cryostat). 


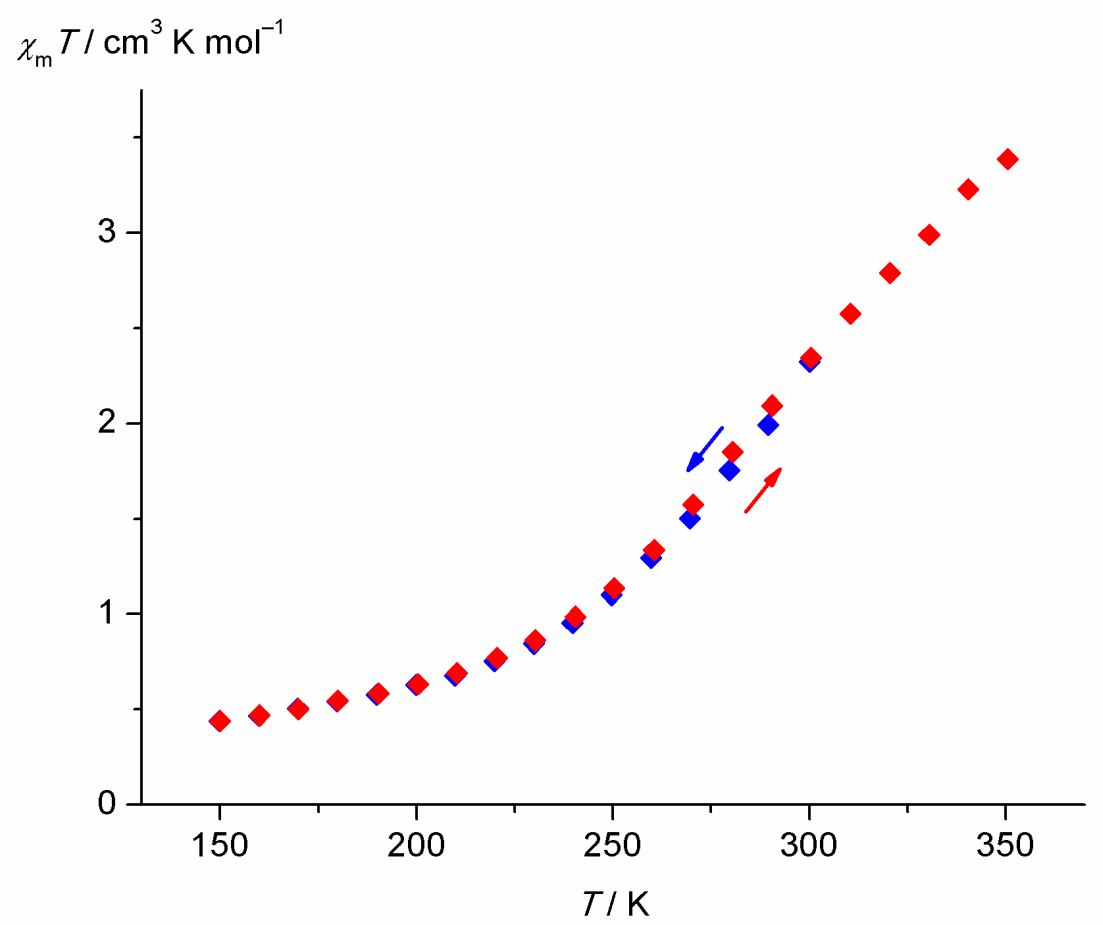

Figure S15. $\chi T$ vs. $T$ data obtained from magnetic susceptibility measurements of 1[OTf] 2 using a SQUID magnetometer during cooling (blue) and re-warming (red).

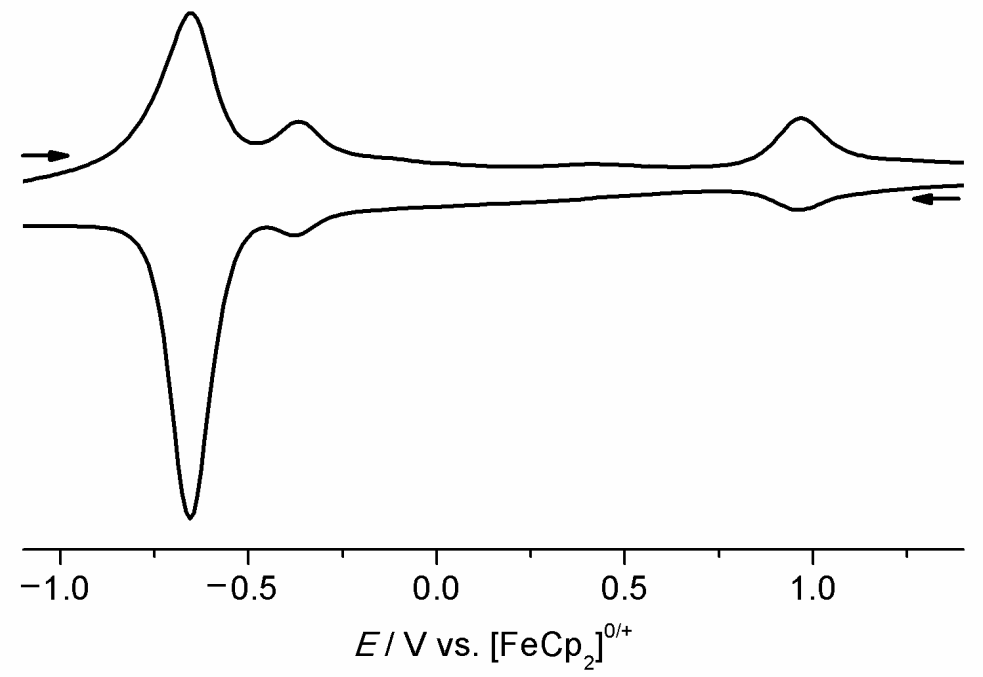

Figure S16. Square-wave voltammograms of 1[OTf]2, $1 \mathrm{mM}$ in acetonitrile containing $0.1 \mathrm{M}$ $\left[{ }^{n} \mathrm{Bu}_{4} \mathrm{~N}\right]\left[\mathrm{PF}_{6}\right]$ as supporting electrolyte. 


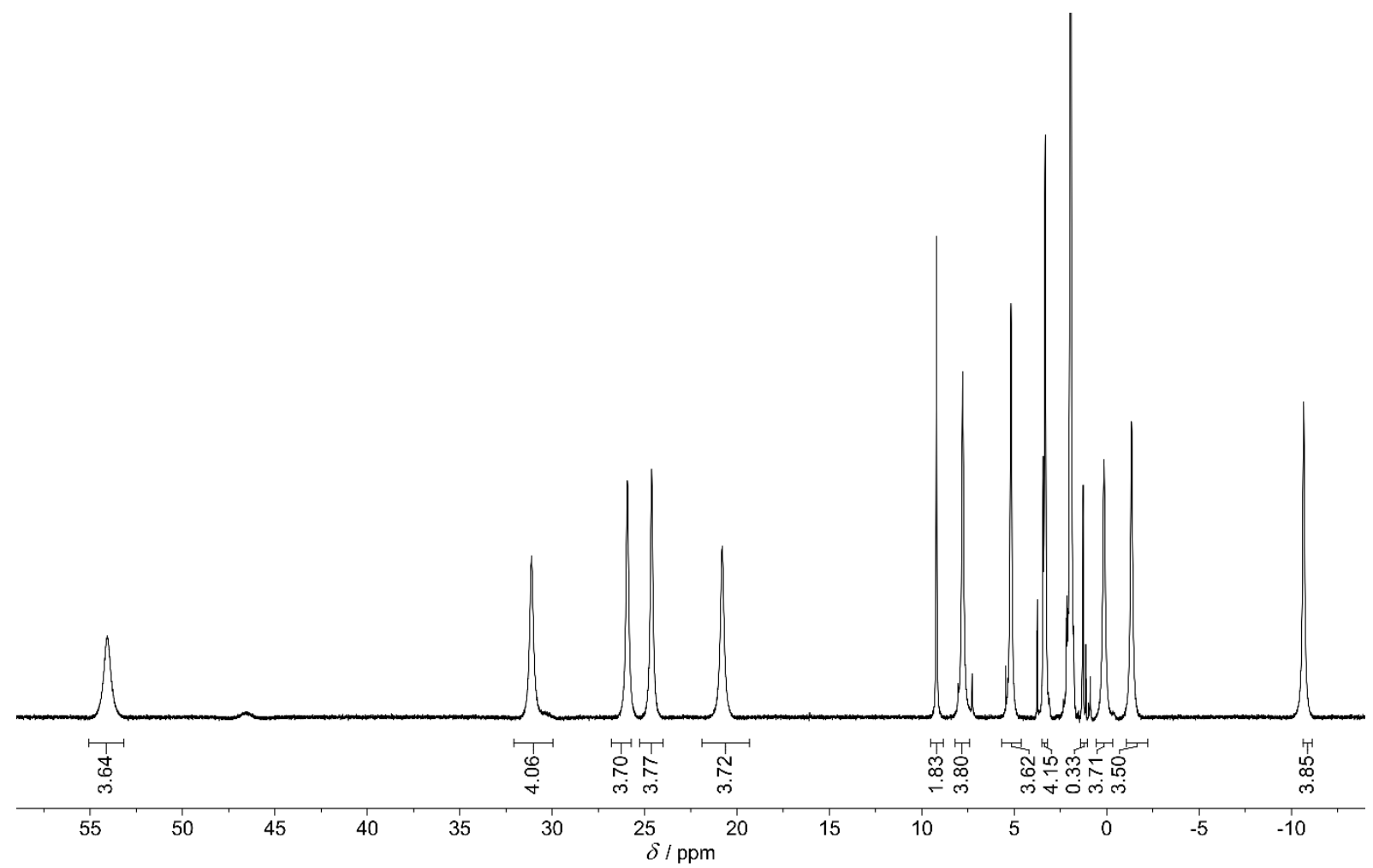

Figure S17. ${ }^{1} \mathrm{H}$ NMR spectrum of $\mathbf{1}[\mathrm{OTF}]_{2}$ after addition of equivalent $\left[\mathrm{FeCp}_{2}\right]\left[\mathrm{PF}_{6}\right]$ in dry, deaerated $\mathrm{CD}_{3} \mathrm{CN}$ at $293 \mathrm{~K}$ (* acetonitrile, ** ferrocene, *** unknown decomposition products).

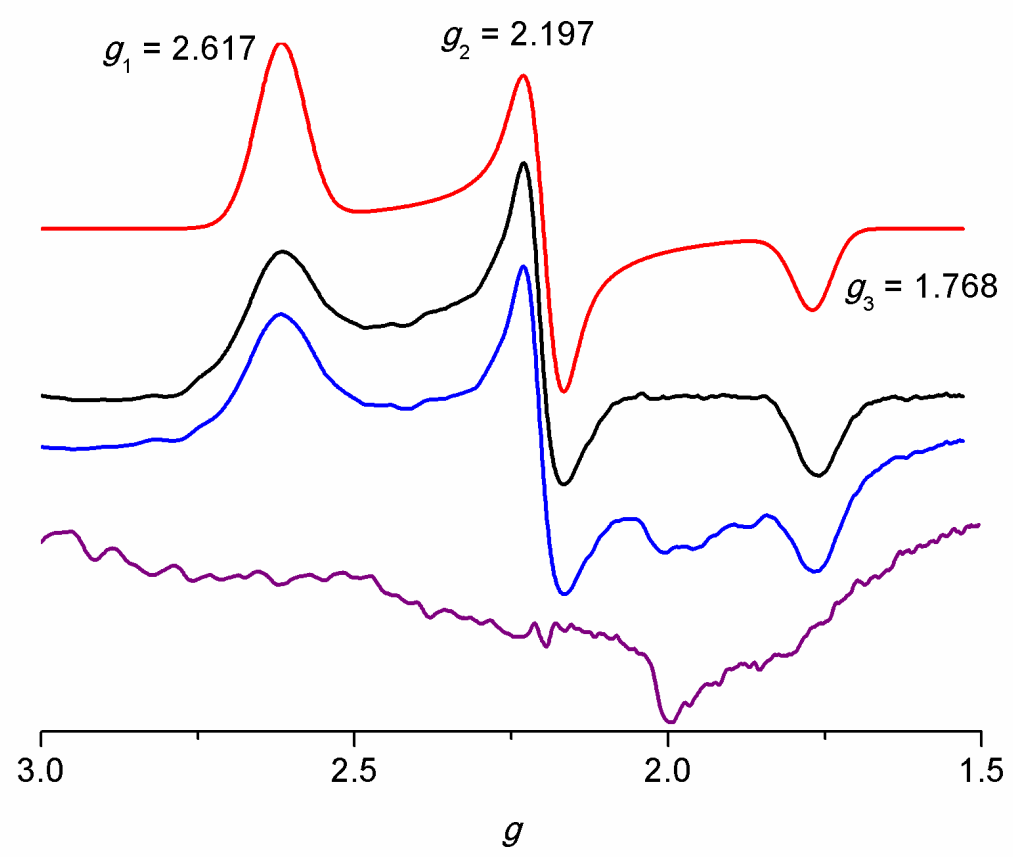

Figure S18. X-band EPR spectrum of $\mathbf{1}[\mathbf{O T f}]_{2}$ with 1 eq of $\left[\mathrm{FeCp}_{2}\right]\left[\mathrm{PF}_{6}\right]$ added in butyronitrile at $77 \mathrm{~K}$ before (blue) and after baseline (purple) correction (black) and simulated spectrum (red). 


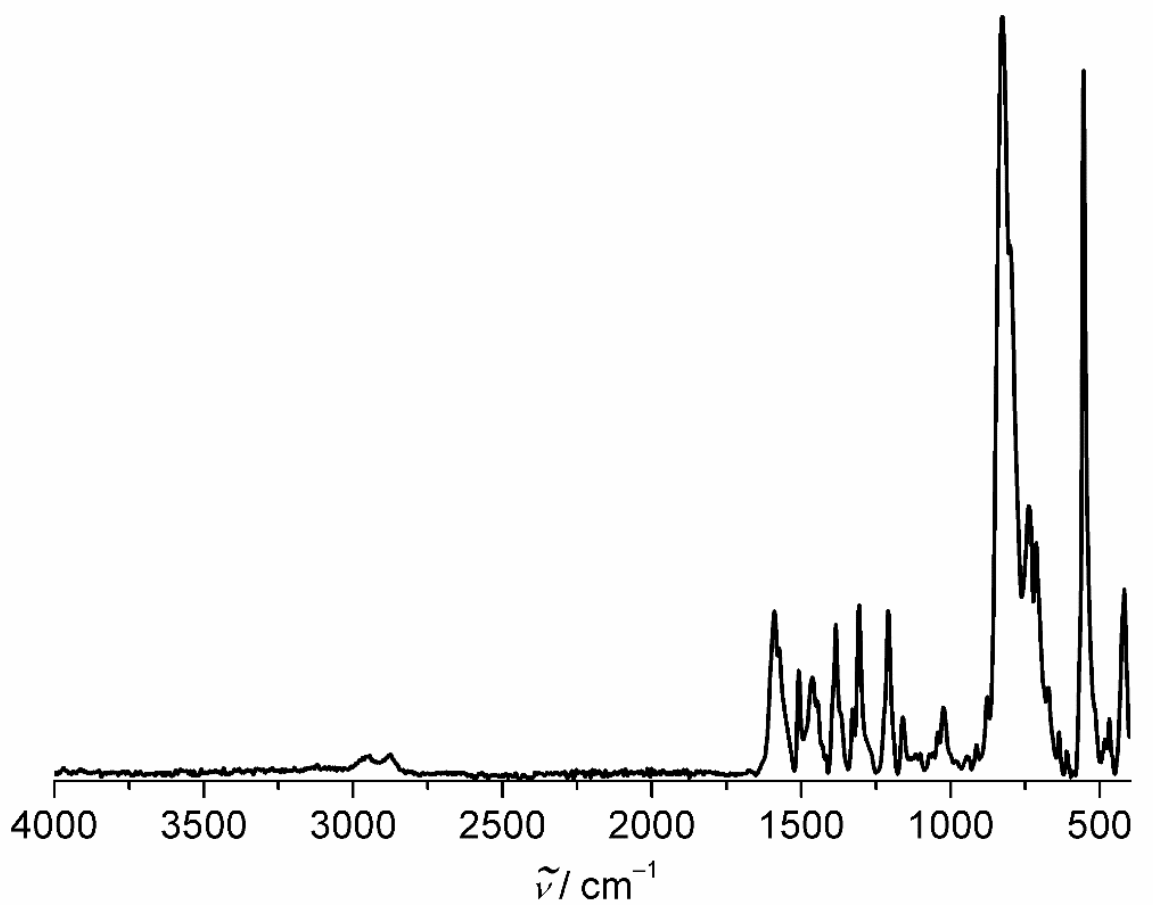

Figure S19. ATR IR spectrum of $\mathbf{1}\left[\mathrm{PF}_{6}\right]_{3}$.

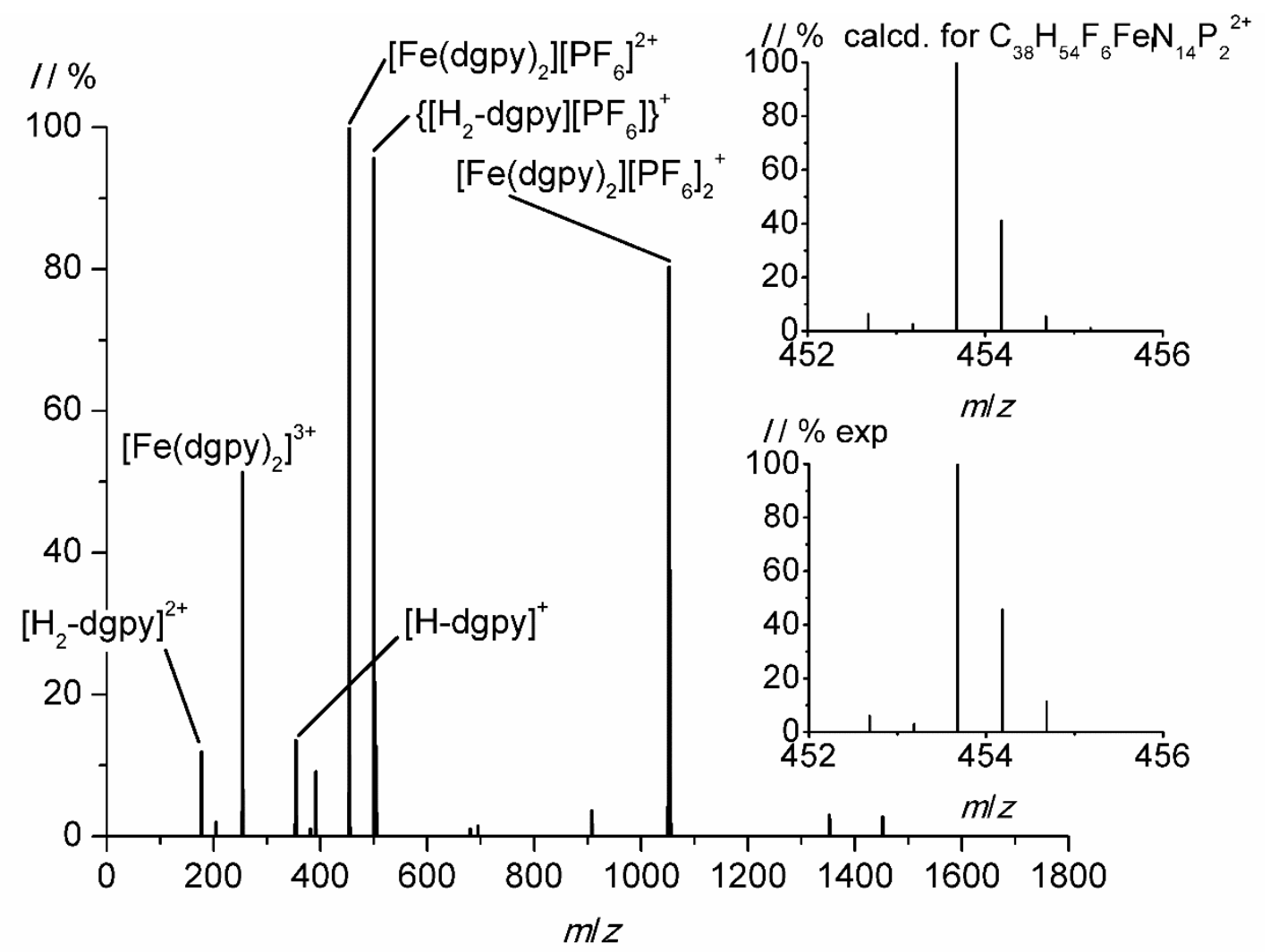

Figure $\mathrm{S20} \mathrm{ESI}^{+}$mass spectrum of $\mathbf{1}\left[\mathrm{PF}_{6}\right]_{3}$ in dry, deaerated acetonitrile. 
a)

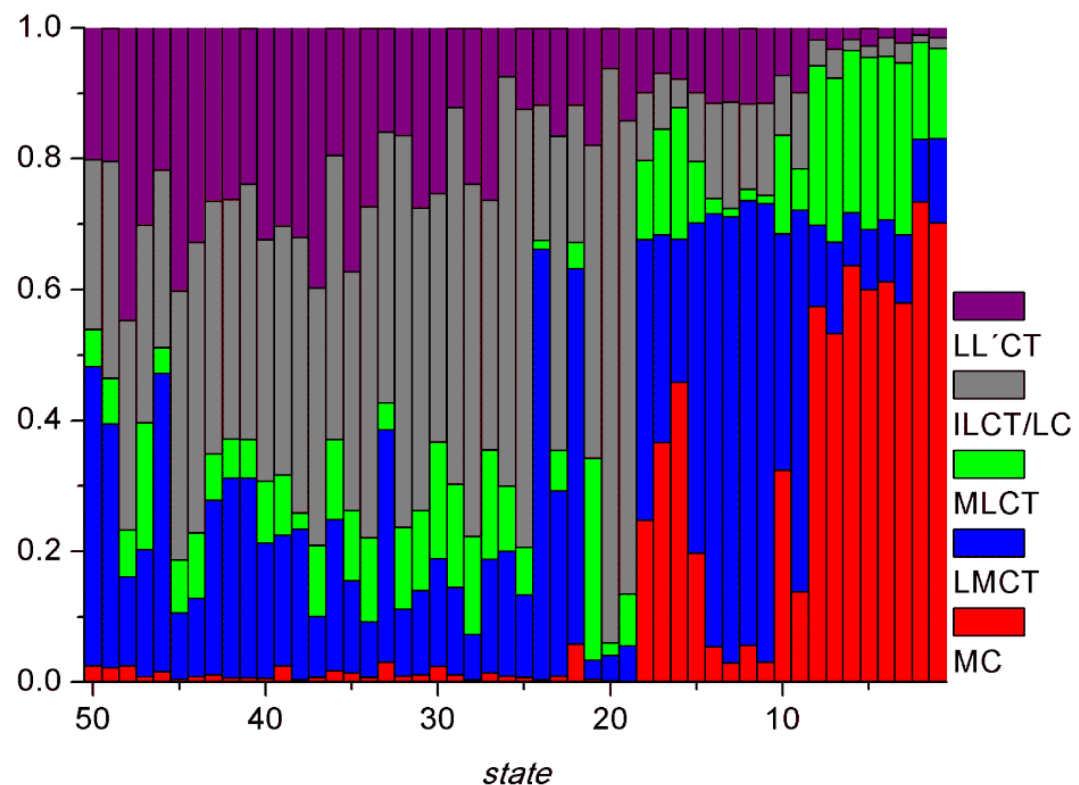

b)
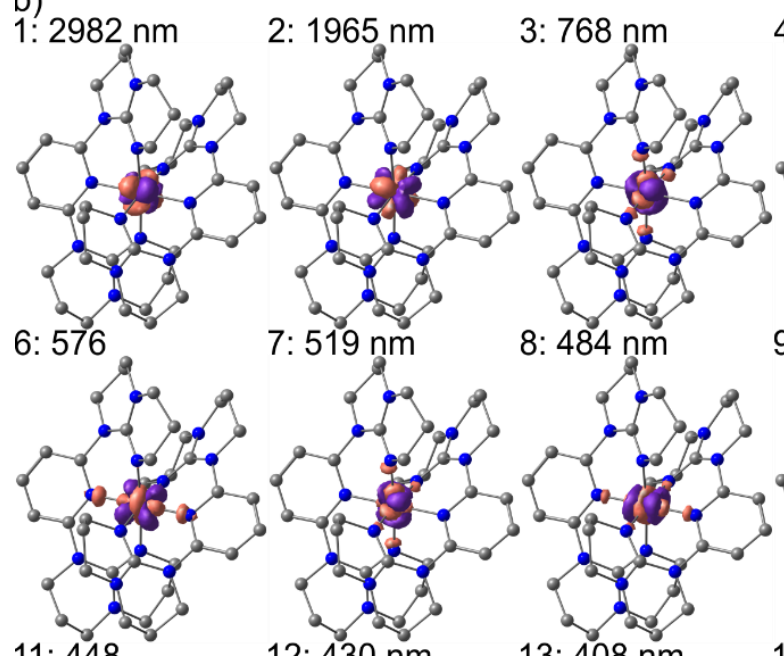

8: $484 \mathrm{~nm}$

11: 448

12: $430 \mathrm{~nm}$
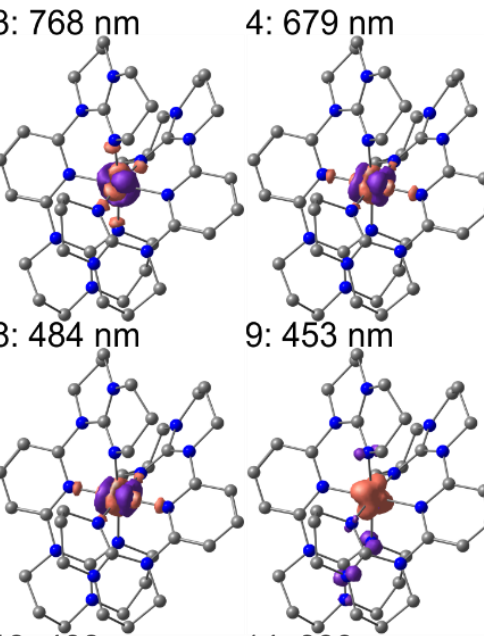

5: $604 \mathrm{~nm}$

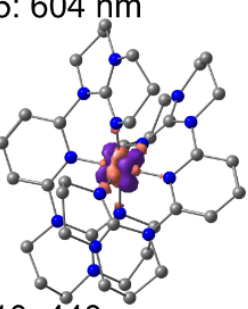

9: $453 \mathrm{~nm}$
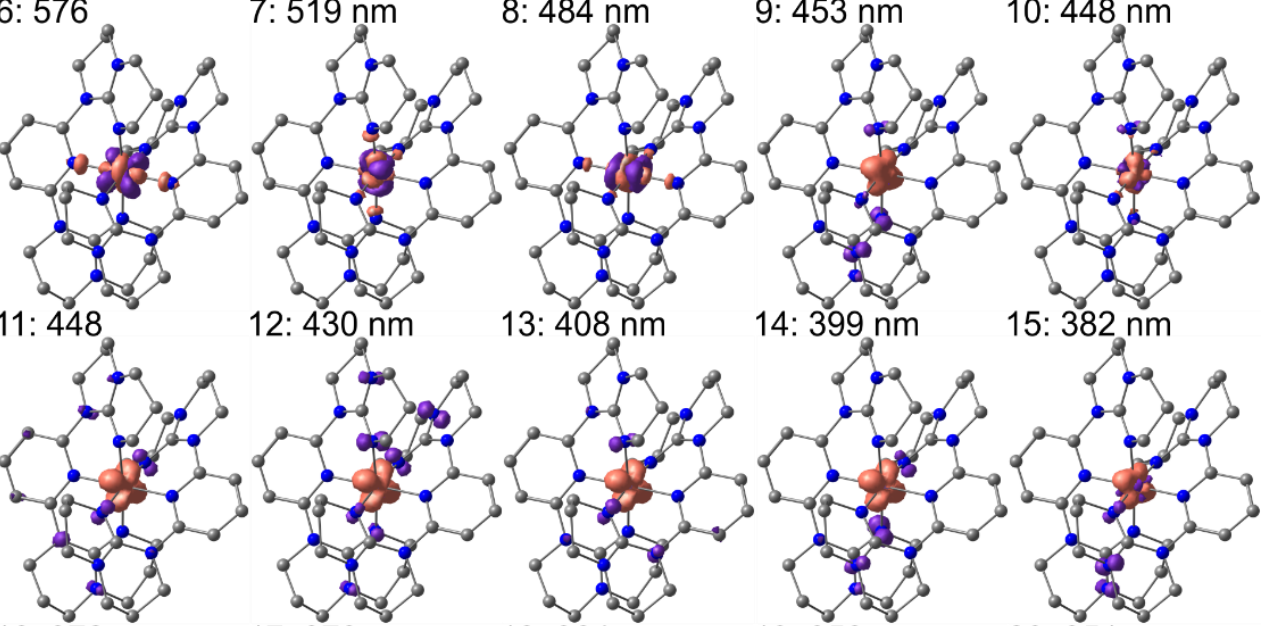

13: $408 \mathrm{~nm}$

14: $399 \mathrm{~nm}$

15: $382 \mathrm{~nm}$
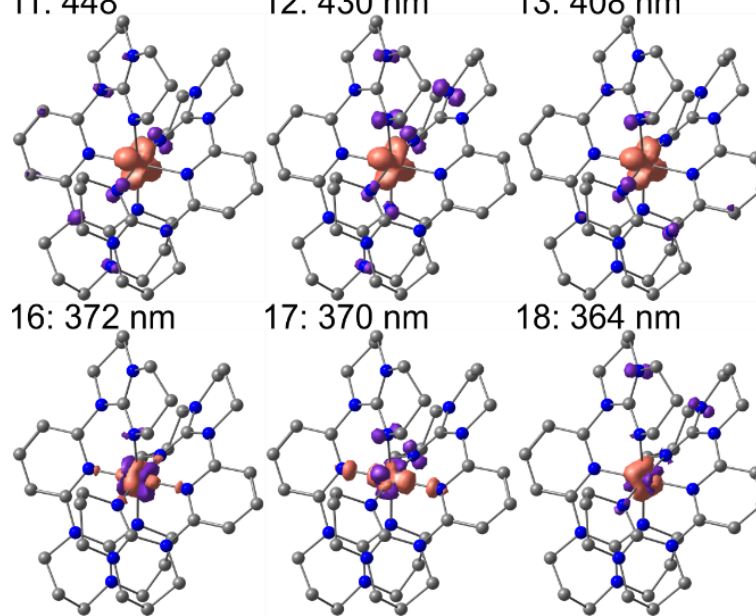

18: $364 \mathrm{~nm}$
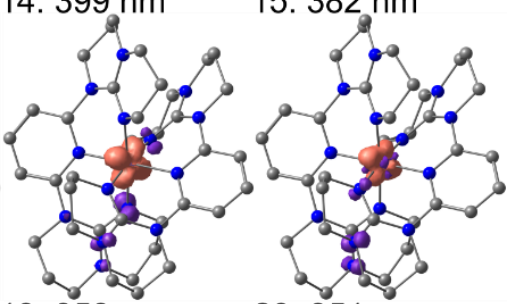

19: $358 \mathrm{~nm}$

20: $351 \mathrm{~nm}$
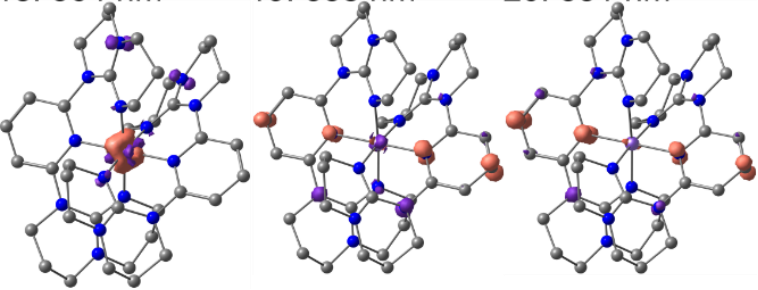

Figure S21. a) Charge transfer analysis of the 50 lowest spin-allowed transitions of low-spin $\mathbf{m e r}$ $\mathbf{1}^{\mathbf{3 +}}$ (red: MC, blue: LMCT, green: MLCT, gray: ILCT/LC, purple: LL'CT) and b) difference densities of the 20 lowest spin-allowed transitions of low-spin $\mathbf{m e r - \mathbf { 1 } ^ { 3 + }}$ with the respective wavelength (purple $=$ electron loss; orange $=$ electron gain). The two lowest transitions reflect the split ${ }^{2} \mathrm{~T}$ ground state. 


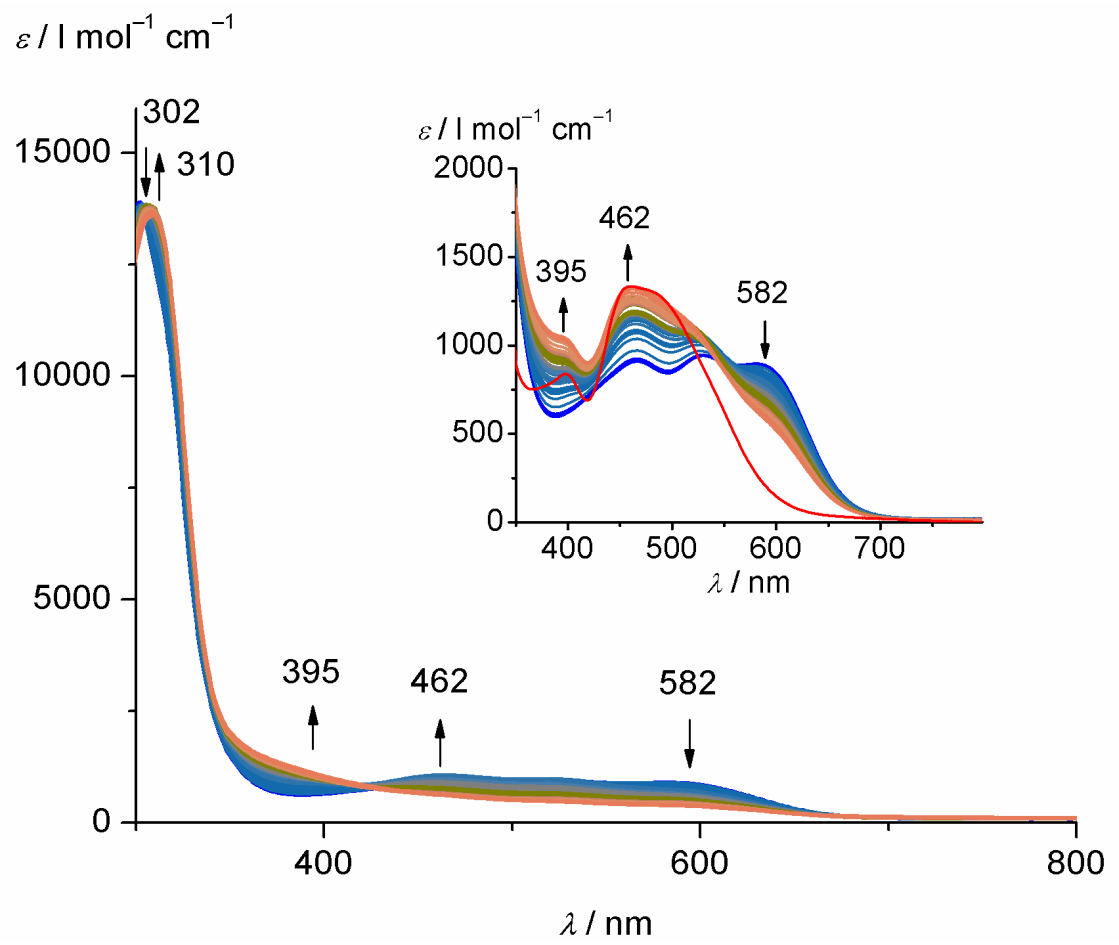

Figure S22. UV/Vis spectra of $\mathbf{1}[\mathbf{O T f}]_{2}$ during electrochemical oxidation in acetonitrile containing $0.1 \mathrm{M}\left[{ }^{n} \mathrm{Bu}_{4} \mathrm{~N}\right]\left[\mathrm{PF}_{6}\right]$ at $300 \mathrm{~K}$.

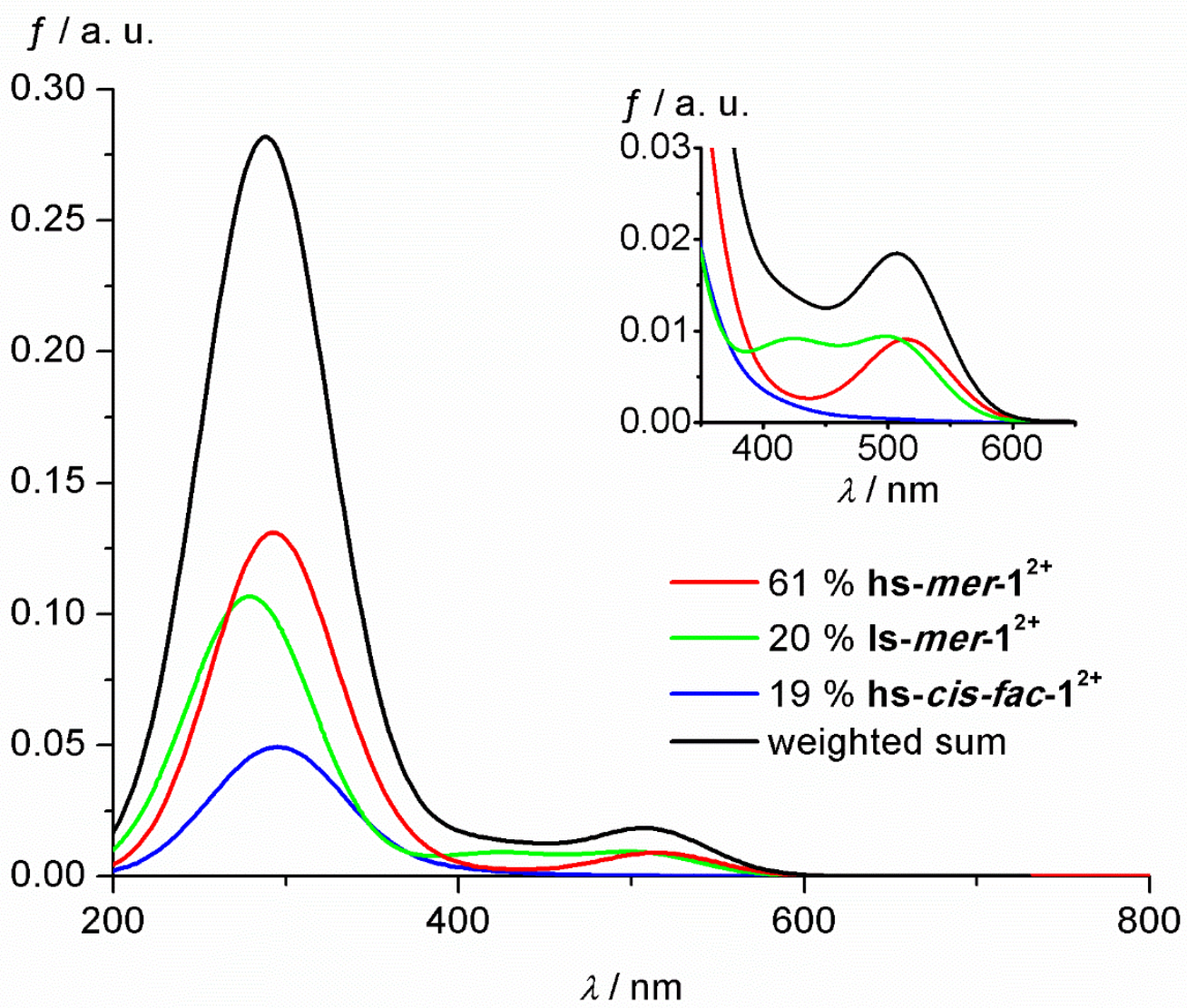

Figure S23. TDDFT simulated UV/Vis spectra of $\mathbf{1}^{\mathbf{2}}$ with Gaussian broadening based on the experimental room temperature composition showing the panchromatic absorption between $400-$ $600 \mathrm{~nm}$. 
mer-1 ${ }^{2+}$, low-spin

$6-1.697611000$

$6 \quad 1.822343000$

$6 \quad 0.746398000$

$6-0.438637000$

$6-0.525854000$

$7 \quad 0.534203000$

$1 \quad 2.736640000$

$1-1.272376000$

$\begin{array}{ll}6 & 1.635715000\end{array}$

$6 \quad 2.341055000$

$6 \quad 3.790134000$

$7 \quad 3.883384000$

$\begin{array}{ll}6 & 2.787313000\end{array}$

$7 \quad 1.717001000$

$1 \quad 4.299199000$

$1 \quad 2.089914000$

$1 \quad 2.276081000$

$6-1.894681000$

$7-3.044793000$

$6-3.097448000$

$6-2.264100000$

$6-0.886409000$

$7-0.918049000$

$1-2.739741000$

$1-0.337083000$

$6 \quad 1.342435000$

$6 \quad 1.270574000$

$6 \quad 0.065022000$

$6-1.046266000$

$6 \quad-0.931579000$

$7 \quad 0.253587000$

$1 \quad 2.132459000$

$1-1.980126000$

$\begin{array}{ll}6 & 2.014117000\end{array}$

$6 \quad 2.671247000$

$6 \quad 4.037895000$

$7 \quad 3.938646000$

$6 \quad 2.778248000$

$7 \quad 1.850501000$

14.474681000

$1 \quad 2.629934000$

$1 \quad 2.766920000$

$\begin{array}{ll}6 & -2.124543000\end{array}$

$7-3.279238000$

$6-3.281629000$

$6-1.865448000$

$6-1.053142000$

$7-1.062209000$

$1-3.868388000$

$1-1.861092000$

$1-0.016263000$

$7 \quad 2.839267000$

$7 \quad-1.776697000$
$-2.801685000$

$-4.191657000$

$-4.956896000$

$-4.335937000$

$-2.945712000$

$-2.187877000$

$-4.670318000$

$-4.927071000$

0.798382000

0.350751000

0.082471000

$-0.732131000$

$-0.950419000$

$-0.222297000$

$-0.464902000$

1.729380000

1.116564000

$-1.228199000$

$-1.108063000$

$-0.183737000$

1.044560000

0.612157000

$-0.375720000$

$-0.689636000$

0.185552000

2.530491000

3.924607000

4.529038000

3.745909000

2.356996000

1.754858000

4.530176000

4.211989000

$-1.039820000$

$-0.442107000$

0.062911000

0.841231000

0.867014000

$-0.029692000$

0.713317000

$-1.865082000$

$-1.183757000$

0.453080000

0.205395000

$-0.686428000$

$-0.965129000$

$-1.347227000$

$-0.281284000$

$-0.199148000$

$-1.768481000$

$-1.548837000$

$-2.048346000$

$-2.350004000$
0.111101000

0.132690000

0.528903000

0.865622000

0.791052000

0.428475000

$-0.169633000$

1.198893000

$-2.164863000$

$-3.430711000$

$-3.083933000$

$-1.871388000$

$-1.084020000$

$-1.122965000$

$-3.882422000$

$-1.812680000$

$-4.203735000$

1.893628000

2.625468000

3.754976000

3.468767000

3.008826000

1.927359000

4.658590000

3.854037000

0.554663000

0.518990000

0.231436000

0.003413000

0.081005000

0.340531000

0.733561000

$-0.253412000$

2.803172000

4.034560000

3.623917000

2.387812000

1.661651000

1.760325000

4.387442000

2.431825000

4.827258000

$-0.907843000$

$-1.597774000$

$-2.757227000$

$-3.212893000$

$-1.992298000$

$-0.993287000$

$-3.540561000$

$-3.949648000$

$-2.249907000$

$-0.213191000$

1.058914000 


\begin{tabular}{|c|c|c|c|}
\hline & -2.941753000 & -3.218603000 & 6000 \\
\hline & -2.949212000 & -4.067048000 & 1.495778000 \\
\hline & -2.851332000 & -3.608896000 & -0.204809000 \\
\hline & 0.830436000 & -6.034760000 & 0.572226000 \\
\hline & -0.008339000 & 5.607467000 & 0.182869000 \\
\hline & 0.393354000 & -0.221002000 & 0.391576000 \\
\hline & -2.102687000 & 1.594944000 & -0.091789000 \\
\hline & 2.603366000 & 1.948208000 & \\
\hline & 3.758908000 & & 6000 \\
\hline & -3.366394000 & & 0000 \\
\hline & -3.542218000 & 3.048274000 & 5000 \\
\hline & -3.279714000 & & \\
\hline & 5.22 & -1.20 & \\
\hline & 5.26 & & -0.1 \\
\hline & 4.12 & -2.72 & -0.0 \\
\hline & 4.10 & -3.17 & \\
\hline & 5.1 & -0.9 & \\
\hline & 5.5 & -1.9 & -2.2 \\
\hline & 5.91 & -0.3 & -1.6 \\
\hline & -4.22 & -1.9 & \\
\hline & -4.22 & -2.4 & \\
\hline & $-5.0^{\prime}$ & -3.1 & \\
\hline & -4.31 & -1.6 & \\
\hline & -4.2 & -2.7 & \\
\hline & -5.09 & -1.3 & \\
\hline & -4.14 & 0.08 & \\
\hline & 4.33 & 1.02 & -2.9 \\
\hline & 1.86 & -0.55 & -3.8 \\
\hline & 0.58 & 1.00 & -2.3 \\
\hline & 4.27 & -3.53 & 000 \\
\hline & 6.21 & -2.2 & \\
\hline & -0.31 & 1.46 & \\
\hline & $-2.1 \xi$ & 1.6 & \\
\hline & -2.74 & 1.6 & \\
\hline & 1.03 & -1.44 & 00 \\
\hline ] & 2.05 & 0.38 & \\
\hline 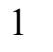 & 4.72 & $-0.7^{\prime}$ & \\
\hline & & & \\
\hline & & & \\
\hline & & & \\
\hline 1 & & & \\
\hline 1 & & & \\
\hline 1 & & & \\
\hline 1 & & & \\
\hline 1 & & & \\
\hline 6 & & & \\
\hline 6 & & & -1.2 \\
\hline 1 & & & \\
\hline 1 & & & \\
\hline 1 & & -0.147204000 & -1.213729000 \\
\hline & & & -1.958878000 \\
\hline & & -1.621613000 & -2.497331000 \\
\hline & -1.433188000 & -0.076074000 & -3.679770000 \\
\hline & -1.459939000 & -2.266551000 & -1.562760000 \\
\hline
\end{tabular}




\begin{tabular}{|c|c|c|c|}
\hline 6 & 1.744217000 & -2.972882000 & 0.201627000 \\
\hline & & -4.358592000 & 0.305076000 \\
\hline & 0.830056000 & -5.123626000 & 0.712963000 \\
\hline & -0.383340000 & -4.519815000 & 0.985187000 \\
\hline & -0.489080000 & -3.138724000 & 0.833421000 \\
\hline & 0.561106000 & -2.389211000 & 0.458618000 \\
\hline & 2.835380000 & -4.836293000 & 0.056806000 \\
\hline & -1.217325000 & -5.111656000 & 1.321380000 \\
\hline 6 & 1.740973000 & 0.588986000 & -2.226578000 \\
\hline 6 & 2.496643000 & 0.121767000 & -3.455829000 \\
\hline & 3.927384000 & -0.148852000 & -3.039045000 \\
\hline 7 & 3.976537000 & & -1.771279000 \\
\hline 6 & 2.839018000 & -1.130476000 & -1.052862000 \\
\hline & 1.752777000 & -0.437204000 & -1.190575000 \\
\hline 1 & 4.454650000 & -0.750884000 & -3.784912000 \\
\hline & 2.199895000 & & -1.845529000 \\
\hline 1 & 2.474724000 & & -4.245226000 \\
\hline 6 & -1.942138000 & -1.449553000 & 1.892242000 \\
\hline 7 & -3.188355000 & & \\
\hline 6 & -3.364725000 & & 073000 \\
\hline & -2.407645000 & & \\
\hline o & -1.0 & & \\
\hline 7 & -0.9 & & \\
\hline 1 & -3.212761000 & & \\
\hline 1 & -0.6 & -0. & \\
\hline 6 & & & \\
\hline 6 & & & \\
\hline 6 & & & \\
\hline 6 & -1.1 & & \\
\hline 6 & -0.9 & & \\
\hline 7 & & & \\
\hline 1 & 2.09 & & \\
\hline 1 & -2.0 & & 5000 \\
\hline 6 & & -0.8 & 5000 \\
\hline 6 & & & 0000 \\
\hline 6 & & & \\
\hline 7 & & & 1000 \\
\hline 6 & & & \\
\hline 7 & & & 7000 \\
\hline 1 & & & 5000 \\
\hline 1 & 0000 & 95000 & 2000 \\
\hline 1 & 3.232070000 & 54000 & 43000 \\
\hline 6 & -2.208903000 & 40278000 & -0.871009000 \\
\hline 7 & -3.396670000 & 0.393695000 & -1.502226000 \\
\hline 6 & -3.446319000 & -0.407790000 & -2.730248000 \\
\hline 6 & -2.052566000 & -0.734962000 & -3.223582000 \\
\hline 6 & -1.229683000 & -1.163794000 & -2.025030000 \\
\hline 7 & -1.153481000 & -0.091036000 & -1.039899000 \\
\hline 1 & -4.008704000 & 0.169219000 & -3.470226000 \\
\hline 1 & -2.100211000 & -1.523799000 & -3.974430000 \\
\hline 1 & -0.212737000 & -1.430496000 & -2.304938000 \\
\hline 7 & 2.873187000 & -2.198773000 & -0.139199000 \\
\hline 7 & -1.758491000 & -2.540304000 & 1.030984000 \\
\hline 6 & -2.898263000 & -3.413011000 & 0.692891000 \\
\hline 1 & -3.004828000 & -4.217927000 & 1.425358000 \\
\hline
\end{tabular}




$\begin{array}{lrrr}1 & -2.687473000 & -3.865443000 & -0.274567000 \\ 1 & 0.939210000 & -6.195288000 & 0.817038000 \\ 1 & -0.065842000 & 5.775794000 & 0.365240000 \\ 26 & 0.375977000 & -0.229765000 & 0.456941000 \\ 7 & -2.152428000 & 1.760474000 & -0.019586000 \\ 7 & 2.573891000 & 2.104975000 & 0.765105000 \\ 6 & 3.704785000 & 2.951256000 & 0.336812000 \\ 6 & -3.412050000 & 2.476824000 & 0.260014000 \\ 1 & -3.612851000 & 3.227615000 & -0.509297000 \\ 1 & -3.291732000 & 2.993722000 & 1.209256000 \\ 6 & 5.308929000 & -1.341452000 & -1.392475000 \\ 6 & 5.289234000 & -1.825524000 & 0.036598000 \\ 6 & 4.172184000 & -2.831400000 & 0.158133000 \\ 1 & 4.114117000 & -3.236126000 & 1.166673000 \\ 1 & 5.124786000 & -0.992923000 & 0.721392000 \\ 1 & 5.641570000 & -2.139602000 & -2.065124000 \\ 1 & 5.995477000 & -0.500698000 & -1.520295000 \\ 6 & -4.371374000 & -2.028726000 & 2.043926000 \\ 6 & -4.181694000 & -2.622090000 & 0.668983000 \\ 1 & -5.010530000 & -3.284761000 & 0.421771000 \\ 1 & -4.136016000 & -1.833927000 & -0.084215000 \\ 1 & -4.565735000 & -2.817470000 & 2.779340000 \\ 1 & -5.222524000 & -1.346144000 & 2.066137000 \\ 1 & -4.399448000 & -0.003060000 & 3.532862000 \\ 1 & 4.479089000 & 0.789688000 & -2.927213000 \\ 1 & 2.032658000 & -0.787964000 & -3.844424000 \\ 1 & 0.702513000 & 0.823880000 & -2.450319000 \\ 1 & 4.370074000 & -3.657549000 & -0.530066000 \\ 1 & 6.236251000 & -2.298175000 & 0.294586000 \\ 1 & -0.312804000 & 1.098624000 & 3.006867000 \\ 1 & -2.455744000 & 1.415564000 & 4.366678000 \\ 1 & -2.694625000 & 1.464738000 & 2.620675000 \\ 1 & 1.239348000 & -1.234244000 & 3.278157000 \\ 1 & 2.480023000 & 0.570548000 & 4.529242000 \\ 1 & 4.987931000 & -0.597740000 & 3.242724000 \\ 6 & 4.973024000 & 2.135782000 & 0.269784000 \\ 6 & 5.256911000 & 1.644292000 & 1.668544000 \\ 1 & 6.080153000 & 0.925276000 & 1.684145000 \\ 1 & 5.790846000 & 2.757779000 & -0.091751000 \\ 1 & 4.850244000 & 1.298422000 & -0.418290000 \\ 1 & 3.863009000 & 3.778771000 & 1.034551000 \\ 1 & 3.446645000 & 3.370908000 & -0.633522000 \\ 1 & 5.543120000 & 2.479173000 & 2.317334000 \\ 6 & -4.570516000 & 1.511870000 & 0.302224000 \\ 6 & -4.694552000 & 0.896832000 & -1.069771000 \\ 1 & -5.482250000 & 2.046083000 & 0.567086000 \\ 1 & -4.396930000 & 0.741103000 & 1.053470000 \\ 1 & -5.396953000 & 0.058528000 & -1.073493000 \\ 1 & -5.064872000 & 1.635812000 & -1.788194000 \\ 1 & -4.010116000 & -1.325363000 & -2.533908000 \\ 1 & -1.593510000 & 0.142588000 & -3.685433000 \\ 1 & -1.679371000 & -2.053894000 & -1.573555000\end{array}$




\begin{tabular}{|c|c|c|c|}
\hline \multicolumn{4}{|c|}{ cis-fac- $\mathbf{1}^{2+}$, low-spin } \\
\hline 26 & 4.375608000 & 2.957099000 & 12.369363000 \\
\hline & 5.950385000 & 2.226160000 & 13.471914000 \\
\hline & 6.887734000 & 0.474884000 & 14.758019000 \\
\hline & 4.702536000 & 0.309116000 & 13.928725000 \\
\hline & 3.251122000 & 2.154832000 & 13.850053000 \\
\hline & 2.017883000 & 4.154014000 & 14.025908000 \\
\hline 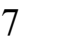 & 2.912867000 & 6.205168000 & 14.684705000 \\
\hline & 4.239294000 & 4.668317000 & 13.481060000 \\
\hline & 2.691682000 & 3.436039000 & 11.283808000 \\
\hline & 3598000 & & 10.314655000 \\
\hline & 4516000 & & 11.379269000 \\
\hline & & & 11.219627000 \\
\hline & & & 10.645534000 \\
\hline & & & 9.356812000 \\
\hline & & & 10.906776000 \\
\hline & 7.184139000 & & 13.643296000 \\
\hline & & & 12.782503000 \\
\hline 1 & & & \\
\hline D & & & \\
\hline 1 & & & \\
\hline 1 & & & \\
\hline 6 & & & \\
\hline 1 & & & \\
\hline 1 & & & \\
\hline 6 & & & \\
\hline 6 & & & \\
\hline 1 & & & 5000 \\
\hline 6 & & & \\
\hline 1 & & & 0000 \\
\hline 1 & & & 5000 \\
\hline 6 & & & 0000 \\
\hline 1 & & & 8000 \\
\hline 1 & & & 3000 \\
\hline 6 & & & 0000 \\
\hline 6 & & & \\
\hline 1 & & -0.8 & 5000 \\
\hline 6 & & & 5000 \\
\hline 1 & & & 9000 \\
\hline 6 & & & 9000 \\
\hline 1 & & & 2000 \\
\hline 6 & & & 75000 \\
\hline 6 & & & 5000 \\
\hline 1 & & & 41000 \\
\hline 1 & & & 22000 \\
\hline 6 & & 5.424456000 & 15.648228000 \\
\hline 1 & & & 16.389978000 \\
\hline 6 & 1.785939000 & 6.532734000 & 15.565726000 \\
\hline 1 & & & 16.549676000 \\
\hline 1 & & 7.431142000 & 15.152300000 \\
\hline 6 & & 5.013648000 & 14.059171000 \\
\hline 6 & 3.871771000 & 7.306454000 & 14.599122000 \\
\hline 1 & & 8.218276000 & 14.380295000 \\
\hline 1 & 4.340349000 & 7.435209000 & 15.579995000 \\
\hline 6 & & 7.037059000 & 13.546385000 \\
\hline 1 & 4.503967000 & 7.214223000 & 12.550976000 \\
\hline
\end{tabular}


5.762189000

5.360137000

5.797947000

6.128079000

2.399911000

2.828265000

2.908774000

0.915275000

0.766039000

0.411310000

1.843523000

$-0.025648000$

0.272297000

0.828329000

1.095500000

0.281382000

3.254642000

3.279327000

2.372293000

4.502642000

4.560631000

5.645640000

6.574335000

5.541087000

7.940637000

8.705915000

8.051229000

8.055376000

8.998043000

7.246741000

7.955559000

7.385745000

8.952705000

6.470662000

7.559074000

8.636236000

7.144493000

6.894007000

7.470901000

6.836225000

5.508024000

4.947467000

4.952690000

0.150430000

0.316342000

$-1.065768000$

$-0.795357000$

0.841564000

$-0.770654000$

0.702507000

$-0.210796000$

7.203848000
7.713978000

5.589409000

5.436673000

5.349360000

4.794062000

5.488968000

4.959753000

5.062803000

6.035712000

5.081091000

2.512697000

0.268835000

$-0.609842000$

0.430120000

$-0.518440000$

1.003300000

0.540213000

$-0.814623000$

$-1.391958000$

$-1.387232000$

$-2.436123000$

$-0.608400000$

$-1.047563000$

0.748804000

1.047530000

1.681923000

0.058289000

1.070825000

0.639531000

0.478537000

2.532872000

2.633822000

2.935738000

2.955485000

4.820449000

4.999974000

5.072157000

5.652959000

5.621942000

6.692128000

5.075279000

5.109950000

5.654985000

1.521393000

3.976740000

0.131018000

1.830710000

1.317716000

4.053862000

4.052693000

5.842973000

$-0.711899000$
13.683846000

13.678020000

14.670287000

12.954517000

10.805445000

11.521731000

9.854458000

10.627311000

10.157152000

11.591972000

10.979792000

10.407693000

9.834859000

11.661811000

12.115860000

12.409417000

11.124270000

10.791078000

10.723533000

10.501635000

10.242828000

10.460807000

10.142822000

10.777570000

10.277976000

10.724185000

10.706361000

8.767075000

8.430819000

8.337695000

8.331505000

7.403120000

8.148559000

10.301589000

9.107774000

9.062141000

8.126407000

10.180936000

11.107425000

9.855697000

10.415958000

9.477110000

11.140597000

9.538979000

9.763130000

10.705141000

9.099873000

8.714419000

9.715991000

8.739255000

15.932033000

16.440376000 


$$
\text { cis-fac- } \mathbf{1}^{2+} \text {, high-spin }
$$

$26 \quad 4.452861000$

$3.089533000 \quad 12.386462000$

5.970892000

2.166675000

13.599507000

$7 \quad 6.813358000$

0.321116000

14.794507000

4.597291000

0.326417000

14.022758000

3.237507000

2.244448000

14.068150000

2.038667000

4.274290000

2.918695000

6.326000000

4.253028000

4.837514000

2.626997000

3.411287000

0.669006000

2.526556000

2.150006000

1.112762000

4.453456000

1.289947000

6.714385000

1.680786000

7.344881000

3.443665000

3.803259000

2.867426000

7.237385000

3.587018000

7.340050000

3.435250000

7.207850000

1.906293000

8.411589000

2.434783000

9.343716000

1.423954000

0.855366000

14.164467000

14.852181000

13.612724000

11.250659000

10.251975000

11.311711000

10.999045000

10.517367000

9.134701000

10.811871000

13.790088000

12.979543000

14.726505000

13.802909000

14.005646000

12.829346000

14.864782000

8.341609000

1.269652000

15.864835000

8.865357000

0.017139000

14.739330000

14.134516000

6.676418000

0.994309000

15.468414000

7.332962000

$-0.976145000$

14.943287000

$-1.508306000 \quad 15.453343000$

5.257857000

$-2.586786000$

15.612555000

4.658045000

$-1.056035000$

16.244730000

4.680980000

$-1.134637000$

14.101610000

3.704079000

$-1.559067000$

13.905456000

5.338099000

$-1.471942000$

13.298804000

14.371157000

14.990096000

2.370790000

0.218156000

15.248619000

2.482361000

$-0.820050000$

0.885217000

15.319157000

0.405980000

0.351476000

15.815743000

$2.237871000 \quad 15.068397000$

1.071105000

2.748813000

15.392713000

$2.902323000 \quad 14.440040000$

$4.929055000 \quad 14.346417000$

2.130611000

5.701056000

13.581737000

0.642164000

4.210902000

14.172123000

$-0.052428000$

5.564164000

15.721629000

16.476228000

15.691267000

6.659228000

16.692198000

6.907153000

15.266847000

7.566630000

14.208771000

3.139749000

5.148849000

14.780650000

3.877765000

7.431510000

14.593465000

3.310991000

8.345972000

15.758405000

4.357974000

7.536466000

13.704790000

7.200727000

12.719238000 
7.891082000

13.844999000

5.374464000

5.756367000

5.847257000

5.581012000

6.121484000

5.530372000

2.280171000

4.752137000

2.686420000

5.468888000

2.776559000

4.936338000

0.780234000

4.938713000

0.560768000

5.903301000

0.297487000

4.919471000

1.853706000

2.433510000

0.106642000

0.096770000

0.460248000

$-0.761691000$

0.313375000

0.958964000

$-0.616662000$

1.265134000

0.875532000

3.368298000

0.505371000

3.447159000

$-0.869502000$

2.574263000

$-1.500628000$

4.697067000

$-1.398094000$

4.799144000

$-2.458866000$

5.815989000

$-0.585112000$

6.764880000

$-1.017733000$

5.646358000

0.788419000

8.037774000

1.151848000

1.825168000

8.784485000

0.182657000

8.182134000

1.118321000

8.140428000

0.707180000

9.094574000

0.480603000

2.555889000

7.986997000

2.595768000

2.985415000

8.970340000

3.032625000

6.542875000

4.866444000

7.524905000

5.042420000

5.091305000

7.027475000

5.733282000

5.715160000

6.764657000

5.175252000

5.206194000

5.768719000

1.356719000

3.825089000

$-0.108972000$

1.619615000

3.795362000

14.768716000

13.039107000

10.771657000

11.482515000

9.815073000

10.614889000

10.155219000

11.592133000

10.922495000

10.371899000

9.800167000

11.618148000

12.085024000

12.361642000

11.025546000

10.787411000

10.782594000

10.522101000

10.332146000

10.443881000

10.180875000

10.653760000

10.163712000

10.584761000

10.626177000

8.651991000

8.322587000

8.255510000

8.154722000

7.220422000

7.957644000

10.151483000

8.833197000

8.687644000

7.884823000

9.940697000

10.814202000

9.594430000

10.319803000

9.448489000

11.096392000

9.496463000

9.748861000

10.675681000

9.087697000

$-0.778789000$

1.183765000

8.650984000

0.867180000

3.829106000

9.742084000

0.5698

3.945935000

8.714457000

$-0.260154000$

5.996688000

15.957621000

7.056683000

$-0.872804000$

16.487153000 
trans-fac- $\mathbf{1}^{2+}$, low-spin

$\begin{array}{lll}6 & 1.643488000 & -2.532894000\end{array}$

$\begin{array}{lll}6 & 1.639530000 & -3.908310000\end{array}$

$\begin{array}{lll}6 & 0.431380000 & -4.572252000\end{array}$

$\begin{array}{lll}6 & -0.750062000 & -3.863368000\end{array}$

$\begin{array}{lll}6 & -0.688482000 & -2.489531000\end{array}$

$\begin{array}{lll}7 & 0.486611000 & -1.859443000\end{array}$

$1 \quad 2.554971000 \quad-4.451433000$

$1-1.689222000 \quad-4.359512000$

$\begin{array}{lll}6 & 2.302363000 & 1.376567000\end{array}$

$\begin{array}{lll}6 & 3.483365000 & 1.255984000\end{array}$

$6 \quad 4.701424000 \quad 0.853388000$

$7 \quad 4.402609000$

$6 \quad 3.110440000$

$7 \quad 2.107010000$

$1 \quad 5.526414000$

$1 \quad 2.427181000$

$1 \quad 3.664273000$

$6-1.921604000$

$\begin{array}{ll}7 & -2.934499000\end{array}$

$6 \quad-3.084999000$

$6-1.736514000$

$6-1.111371000$

$7-1.026566000$

$1-3.782438000$

$1-1.716769000$

$\begin{array}{ll}6 & 1.635576000\end{array}$

$6 \quad 1.631856000$

$6 \quad 0.415606000$

$\begin{array}{ll}6 & -0.757602000\end{array}$

$6-0.688593000$

$7 \quad 0.477140000$

$1 \quad 2.542453000$

$1-1.692423000$

$6 \quad 2.216152000$

$6 \quad 2.515567000$

$6 \quad 3.838963000$

$7 \quad 3.936773000$

$6 \quad 2.899819000$

$7 \quad 2.008928000$

$1 \quad 3.969938000$

$1 \quad 3.071660000$

$1 \quad 2.591597000$

$6-1.844839000$

$\begin{array}{ll}7 & -2.899669000\end{array}$

$6 \quad-3.069417000$

$6-2.475853000$

$6-1.053792000$

$7-0.970875000$

$1-4.135881000$

$1-2.480376000$

$1-0.645070000$

$7 \quad 2.882954000$

$7-1.863455000$

$6 \quad-3.154150000$

$1-3.773444000$
$-0.314085000$

$-0.614025000$

0.179237000

0.577773000

2.249626000

2.198818000

$-0.489337000$

$-0.337743000$

0.929696000

1.597807000

1.700967000

0.405901000

1.582915000

2.377926000

2.676878000

4.072853000

4.733423000

4.026197000

2.631848000

1.981766000

4.640174000

4.538373000

$-1.389436000$

$-1.046247000$

$-0.306758000$

0.591473000

0.749028000

$-0.170175000$

0.292995000

$-1.934236000$

0.633331000

0.526584000

$-0.683326000$

$-1.866977000$

$-0.280703000$

$-0.821624000$

$-2.743727000$

$-2.326256000$

$-1.874074000$

$-1.733618000$

$-2.403139000$

$-1.750855000$
$-1.942436000$

$-1.528401000$
$-0.922980000$

$-1.167316000$

$-1.263777000$

$-1.190941000$

$-0.955679000$

$-0.719999000$

$-1.317954000$

$-1.367762000$

$-2.438069000$

$-3.383295000$

$-2.586722000$

$-1.756971000$

$-1.425299000$

$-1.633220000$

$-3.245795000$

$-1.788787000$

$-3.900431000$

$-1.605003000$

$-2.507691000$

$-3.224028000$

$-3.383177000$

$-2.008652000$

$-1.332585000$

$-2.687852000$

$-1.403594000$

0.803599000

0.719309000

0.784262000

0.962889000

0.963556000

0.776197000

0.653758000

1.122415000

2.202455000

3.652530000

3.653145000

2.496563000

1.619801000

1.425503000

4.558807000

1.796708000

4.267878000

1.799428000

2.663486000

3.462066000

2.730539000

2.311389000

1.538678000

3.645477000

3.378526000

1.695181000

$-0.837299000$

$-0.944855000$

$-0.755237000$

$-0.134685000$ 


$\begin{array}{lrrr}1 & -2.996133000 & -3.320596000 & -0.198699000 \\ 1 & 0.414308000 & -5.635062000 & -1.465866000 \\ 1 & 0.394899000 & 5.815159000 & 0.756671000 \\ 26 & 0.462198000 & 0.042703000 & 0.070366000 \\ 7 & -1.832925000 & 1.870587000 & 1.137520000 \\ 7 & 2.835698000 & 1.977556000 & 0.920176000 \\ 6 & 4.070514000 & 2.763003000 & 0.743310000 \\ 6 & -3.171580000 & 2.413388000 & 0.871905000 \\ 1 & -3.711832000 & 1.643917000 & 0.318461000 \\ 1 & -3.082054000 & 3.276571000 & 0.221218000 \\ 6 & 5.580744000 & -1.065590000 & -1.338951000 \\ 6 & 5.241722000 & -1.927894000 & -0.150702000 \\ 6 & 4.027981000 & -2.745135000 & -0.517772000 \\ 1 & 3.727912000 & -3.395575000 & 0.300524000 \\ 1 & 5.043985000 & -1.310483000 & 0.727662000 \\ 1 & 5.954832000 & -1.677228000 & -2.166958000 \\ 1 & 6.359476000 & -0.340629000 & -1.093152000 \\ 6 & -4.063222000 & -1.252996000 & -2.701600000 \\ 6 & -3.828339000 & -2.619317000 & -2.093719000 \\ 1 & -4.782108000 & -3.134144000 & -1.977514000 \\ 1 & -3.190062000 & -3.225407000 & -2.738415000 \\ 1 & -4.951793000 & -0.795081000 & -2.251777000 \\ 1 & -4.247280000 & -1.329389000 & -3.774249000 \\ 1 & -3.529895000 & 0.701140000 & -4.192499000 \\ 1 & 5.050911000 & 1.680754000 & -1.959000000 \\ 1 & 3.271102000 & 0.492564000 & -4.136415000 \\ 1 & 1.397789000 & 1.546147000 & -3.018330000 \\ 1 & 4.281305000 & -3.378361000 & -1.373380000 \\ 1 & 6.067281000 & -2.598527000 & 0.085270000 \\ 1 & -0.118755000 & 2.133969000 & -2.045816000 \\ 1 & -1.858449000 & 2.588112000 & -3.822312000 \\ 1 & -1.104701000 & 1.004404000 & -4.048635000 \\ 1 & 1.350974000 & -2.027830000 & 2.094373000 \\ 1 & 1.714198000 & -0.422557000 & 4.054760000 \\ 1 & 4.668847000 & -1.018508000 & 3.604329000 \\ 6 & 5.296145000 & 1.927127000 & 1.021138000 \\ 6 & 5.167323000 & 1.364110000 & 2.417227000 \\ 1 & 5.992393000 & 0.688292000 & 2.654636000 \\ 1 & 6.181853000 & 2.554665000 & 0.932776000 \\ 1 & 5.387722000 & 1.112144000 & 0.303222000 \\ 1 & 4.075151000 & 3.611934000 & 1.430672000 \\ 1 & 4.076382000 & 3.154310000 & -0.274148000 \\ 1 & 5.173830000 & 2.168940000 & 3.161677000 \\ 6 & -3.913254000 & 2.731257000 & 2.167734000 \\ 6 & -3.428896000 & 1.763095000 & 3.251692000 \\ 1 & -4.985113000 & 2.626338000 & 1.993958000 \\ 1 & -3.725434000 & 3.755750000 & 2.491818000 \\ 1 & -2.652508000 & 2.233926000 & 3.863139000 \\ 1 & -4.241022000 & 1.479074000 & 3.916466000 \\ 1 & -2.578046000 & -0.553306000 & 4.433171000 \\ 1 & -3.078466000 & -2.104056000 & 1.855066000 \\ 1 & -0.422518000 & -1.448650000 & 3.199662000\end{array}$


trans-fac- $\mathbf{1}^{2+}$, high-spin

$\begin{array}{lll}6 & 1.671062000 & -2.609604000\end{array}$

$\begin{array}{lll}6 & 1.673979000 & -3.991629000\end{array}$

$\begin{array}{lll}6 & 0.458492000 & -4.644831000\end{array}$

$\begin{array}{lll}6 & -0.727273000 & -3.936779000\end{array}$

$\begin{array}{lll}6 & -0.660299000 & -2.556495000\end{array}$

7

1

1

6

6

6

7

6

$1-0.785524000$

$7 \quad 2.897039000$

$7-1.830029000$

$6-3.105048000$

$1 \quad-3.686998000$
$-1.936718000$

$-4.546108000$

$-4.441107000$

1.390394000

1.322732000

0.905115000

$-0.307327000$

$-0.639948000$

0.155928000

0.677013000

2.236887000

2.288146000

$-0.543609000$

$-0.397733000$

0.872850000

1.576943000

1.669573000

0.369154000

1.501625000

2.330478000

2.713867000

4.106279000

4.760461000

4.063622000

2.670256000

2.025648000

4.677703000

4.587650000

$-1.396559000$

$-1.112139000$

$-0.379725000$

0.598324000

0.767681000

$-0.151174000$

0.158297000

$-1.965761000$

$-2.031348000$

0.665783000

0.615081000

$-0.583939000$

$-1.798196000$

$-1.534278000$

$-0.287836000$

$-0.666710000$

$-2.677056000$

$-2.348063000$

$-1.931850000$

$-1.790018000$

$-2.453412000$

$-1.783970000$
$-1.045968000$

$-1.255974000$

$-1.360954000$

$-1.308463000$

$-1.104921000$

$-0.905861000$

$-1.370543000$

$-1.472435000$

$-2.380536000$

$-3.299970000$

$-2.485821000$

$-1.724389000$

$-1.439797000$

$-1.626732000$

$-3.128123000$

$-1.692779000$

$-3.773144000$

$-1.730747000$

$-2.527056000$

$-3.216194000$

$-3.488618000$

$-2.179375000$

$-1.533089000$

$-2.614563000$

$-1.501926000$

0.872957000

0.719699000

0.715474000

0.877693000

0.958154000

0.875202000

0.647555000

0.963794000

2.192308000

3.648902000

3.664212000

2.572724000

1.690945000

1.454954000

4.604348000

1.757361000

4.228934000

1.802713000

2.712114000

3.523416000

2.762443000

2.278701000

1.512268000

3.761432000

3.407251000

1.639415000

$-0.926831000$

$-1.077207000$

$-0.787547000$

$-0.149633000$ 


$\begin{array}{lrrr}1 & -2.909755000 & -3.354433000 & -0.215931000 \\ 1 & 0.439001000 & -5.711490000 & -1.542882000 \\ 1 & 0.427763000 & 5.839640000 & 0.633110000 \\ 26 & 0.518456000 & 0.045033000 & -0.061862000 \\ 7 & -1.799741000 & 1.898609000 & 1.116490000 \\ 7 & 2.872342000 & 2.019007000 & 1.032479000 \\ 6 & 4.104385000 & 2.813813000 & 0.870117000 \\ 6 & -3.138816000 & 2.454146000 & 0.878766000 \\ 1 & -3.709342000 & 1.675147000 & 0.372368000 \\ 1 & -3.061606000 & 3.292704000 & 0.194681000 \\ 6 & 5.590315000 & -1.054202000 & -1.292627000 \\ 6 & 5.224331000 & -1.950479000 & -0.138471000 \\ 6 & 4.044250000 & -2.785315000 & -0.569250000 \\ 1 & 3.722139000 & -3.449633000 & 0.229588000 \\ 1 & 4.976364000 & -1.352694000 & 0.740917000 \\ 1 & 5.996197000 & -1.639278000 & -2.124581000 \\ 1 & 6.350950000 & -0.325715000 & -1.004815000 \\ 6 & -4.133208000 & -1.358598000 & -2.703582000 \\ 6 & -3.854931000 & -2.708765000 & -2.076604000 \\ 1 & -4.796798000 & -3.225746000 & -1.892395000 \\ 1 & -3.250037000 & -3.328980000 & -2.739628000 \\ 1 & -5.029750000 & -0.918837000 & -2.253563000 \\ 1 & -4.323581000 & -1.455303000 & -3.773876000 \\ 1 & -3.800968000 & 0.640986000 & -4.144490000 \\ 1 & 5.027646000 & 1.706387000 & -1.806804000 \\ 1 & 3.336547000 & 0.587562000 & -4.088432000 \\ 1 & 1.419409000 & 1.572432000 & -2.970790000 \\ 1 & 4.340039000 & -3.404434000 & -1.421416000 \\ 1 & 6.054133000 & -2.607232000 & 0.119758000 \\ 1 & -0.238476000 & 2.122887000 & -2.319179000 \\ 1 & -2.156827000 & 2.569975000 & -3.897862000 \\ 1 & -1.388613000 & 1.009420000 & -4.220522000 \\ 1 & 1.352047000 & -2.001410000 & 2.080737000 \\ 1 & 1.780723000 & -0.498327000 & 4.087479000 \\ 1 & 4.723914000 & -1.089047000 & 3.553314000 \\ 6 & 5.336142000 & 1.991356000 & 1.159708000 \\ 6 & 5.186895000 & 1.399715000 & 2.541907000 \\ 1 & 6.020601000 & 0.737819000 & 2.786230000 \\ 1 & 6.213303000 & 2.634279000 & 1.100035000 \\ 1 & 5.457352000 & 1.189917000 & 0.429786000 \\ 1 & 4.089788000 & 3.662786000 & 1.557387000 \\ 1 & 4.120392000 & 3.206794000 & -0.146879000 \\ 1 & 5.156848000 & 2.189287000 & 3.301793000 \\ 6 & -3.829080000 & 2.827026000 & 2.188567000 \\ 6 & -3.329993000 & 1.876845000 & 3.283094000 \\ 1 & -4.908570000 & 2.743576000 & 2.054632000 \\ 1 & -3.605954000 & 3.855033000 & 2.476596000 \\ 1 & -2.525243000 & 2.346860000 & 3.856613000 \\ 1 & -4.125928000 & 1.628811000 & 3.981097000 \\ 1 & -2.488810000 & -0.479780000 & 4.467644000 \\ 1 & -3.203051000 & -1.991081000 & 1.915427000 \\ 1 & -0.453592000 & -1.500941000 & 3.139587000\end{array}$




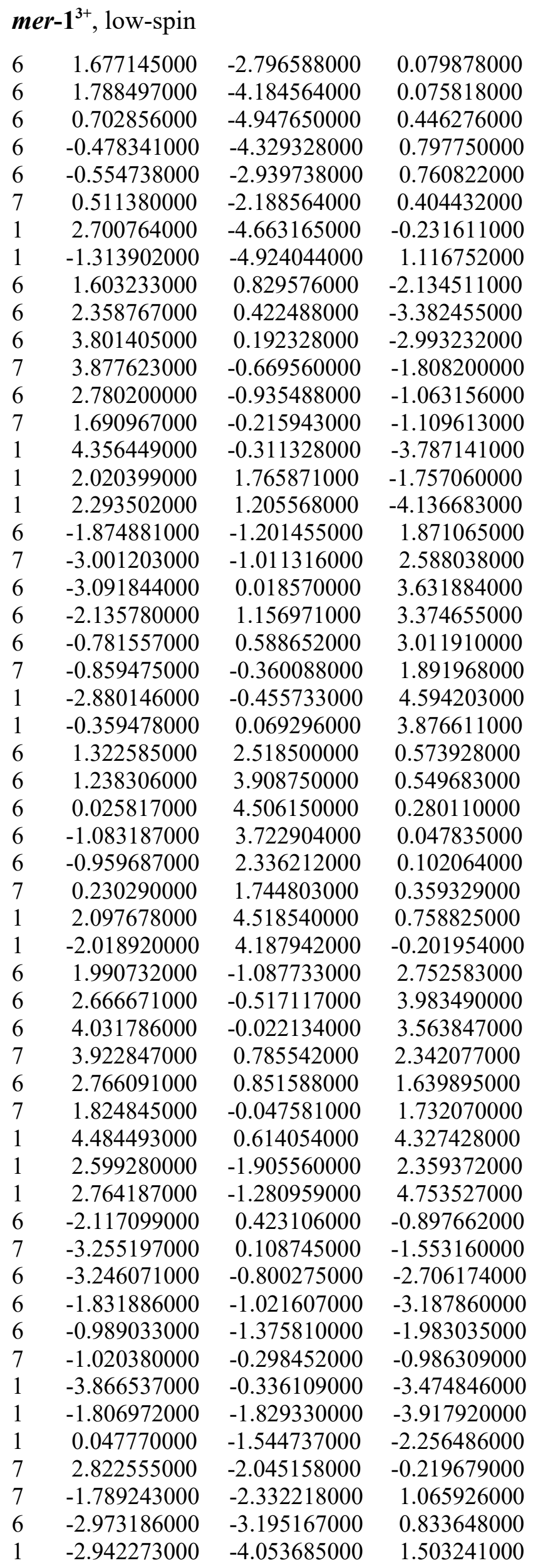




$\begin{array}{lrrr}1 & -2.914604000 & -3.556467000 & -0.191696000 \\ 1 & 0.776965000 & -6.026265000 & 0.461015000 \\ 1 & -0.053801000 & 5.583954000 & 0.244794000 \\ 26 & 0.367015000 & -0.225173000 & 0.385342000 \\ 7 & -2.117830000 & 1.566823000 & -0.104093000 \\ 7 & 2.585236000 & 1.941585000 & 0.784792000 \\ 6 & 3.737734000 & 2.818901000 & 0.487163000 \\ 6 & -3.394299000 & 2.291007000 & 0.087387000 \\ 1 & -3.550675000 & 3.001646000 & -0.726876000 \\ 1 & -3.318111000 & 2.849133000 & 1.016137000 \\ 6 & 5.211995000 & -1.197727000 & -1.524083000 \\ 6 & 5.254048000 & -1.760296000 & -0.124632000 \\ 6 & 4.110994000 & -2.734722000 & 0.004817000 \\ 1 & 4.072432000 & -3.180383000 & 0.996872000 \\ 1 & 5.170726000 & -0.966481000 & 0.618749000 \\ 1 & 5.471551000 & -1.966158000 & -2.258083000 \\ 1 & 5.917040000 & -0.372994000 & -1.646759000 \\ 6 & -4.161358000 & -1.901572000 & 2.504712000 \\ 6 & -4.254079000 & -2.452584000 & 1.102947000 \\ 1 & -5.087710000 & -3.147168000 & 1.011444000 \\ 1 & -4.409131000 & -1.646737000 & 0.385956000 \\ 1 & -4.078264000 & -2.708920000 & 3.238415000 \\ 1 & -5.034790000 & -1.305564000 & 2.761536000 \\ 1 & -4.122018000 & 0.371046000 & 3.654663000 \\ 1 & 4.304513000 & 1.140880000 & -2.782861000 \\ 1 & 1.925445000 & -0.488469000 & -3.802192000 \\ 1 & 0.552465000 & 1.007003000 & -2.346094000 \\ 1 & 4.251558000 & -3.532413000 & -0.727224000 \\ 1 & 6.193398000 & -2.283281000 & 0.046564000 \\ 1 & -0.089875000 & 1.376694000 & 2.745290000 \\ 1 & -2.057792000 & 1.774533000 & 4.269088000 \\ 1 & -2.507662000 & 1.788240000 & 2.570615000 \\ 1 & 1.013950000 & -1.496089000 & 2.987813000 \\ 1 & 2.070982000 & 0.302204000 & 4.390887000 \\ 1 & 4.712874000 & -0.858325000 & 3.382117000 \\ 6 & 5.025131000 & 2.039712000 & 0.575414000 \\ 6 & 5.138976000 & 1.515151000 & 1.985893000 \\ 1 & 5.973633000 & 0.818852000 & 2.091251000 \\ 1 & 5.860039000 & 2.699798000 & 0.346085000 \\ 1 & 5.035752000 & 1.224672000 & -0.148703000 \\ 1 & 3.787511000 & 3.643838000 & 1.201074000 \\ 1 & 3.582226000 & 3.231656000 & -0.507746000 \\ 1 & 5.301300000 & 2.336056000 & 2.690703000 \\ 6 & -4.546689000 & 1.320768000 & 0.117151000 \\ 6 & -4.582706000 & 0.630590000 & -1.222455000 \\ 1 & -5.473237000 & 1.865320000 & 0.289790000 \\ 1 & -4.422613000 & 0.601129000 & 0.927245000 \\ 1 & -5.269941000 & -0.217925000 & -1.223898000 \\ 1 & -4.907832000 & 1.321082000 & -2.005116000 \\ 1 & -3.715925000 & -1.746168000 & -2.421312000 \\ 1 & -1.443987000 & -0.119157000 & -3.666197000 \\ 1 & -1.364130000 & -2.304608000 & -1.548606000\end{array}$

